\title{
Increased Dynamics of $\alpha$-Synuclein Fibrils by $\beta$-Synuclein Leads to Reduced Seeding and Cytotoxicity
}

\begin{abstract}
Xue Yang ${ }^{1,3}$, Jonathan K. Williams ${ }^{10}{ }^{1,3}$, Run Yan' ${ }^{2}$, M. Maral Mouradian² \& Jean Baum ${ }^{1 *}$
Alpha-synuclein $(\alpha S)$ fibrils are toxic to cells and contribute to the pathogenesis and progression of Parkinson's disease and other synucleinopathies. $\beta$-Synuclein ( $\beta S$ ), which co-localizes with $\alpha \mathrm{S}$, has been shown to provide a neuroprotective effect, but the molecular mechanism by which this occurs remains elusive. Here we show that $\alpha S$ fibrils formed in the presence of $\beta S$ are less cytotoxic, exhibit reduced cell seeding capacity and are more resistant to fibril shedding compared to $\alpha \mathrm{S}$ fibrils alone. Using solid-state NMR, we found that the overall structure of the core of $\alpha \mathrm{S}$ fibrils when co-incubated with $\beta S$ is minimally perturbed, however, the dynamics of Lys and Thr residues, located primarily in the imperfect KTKEGV repeats of the $\alpha S \mathrm{~N}$-terminus, are increased. Our results suggest that amyloid fibril dynamics may play a key role in modulating toxicity and seeding. Thus, enhancing the dynamics of amyloid fibrils may be a strategy for future therapeutic targeting of neurodegenerative diseases.
\end{abstract}

Parkinson's disease (PD) is a progressively debilitating neurodegenerative disorder that is estimated to affect $1 \%$ of the world's population over the age of $60^{1}$. Amyloid fibril deposits of the protein alpha-synuclein $(\alpha S)$ are found in Lewy bodies (LB) and Lewy neurites $(\mathrm{LN})^{2,3}$ in the substantia nigra and other brain regions of PD patients. Myriad evidence shows that $\alpha S$ fibrils are toxic to cells ${ }^{4-7}$, yet the precise role of $\alpha S$ in the pathology of PD and other synucleinopathies is still unclear.

Several hypotheses have been proposed to explain the experimentally observed cellular toxicity of the fibrils. Among these, seeding-propagation is a proposed mechanism to explain the observed cytotoxicity of the fibrils and the progressive nature of the disease. This process involves the release of mature $\alpha \mathrm{S}$ amyloid seeds from the cell $^{8-10}$ that can then be taken up by a neighboring cell ${ }^{10,11}$; these seeds then template the further misfolding and aggregation of endogenous monomeric $\alpha \mathrm{S}$ in the recipient $\mathrm{cell}^{7,12}$. Fibril polymorphism and protofilament packing have been shown to play an important role in seeding capacity and toxicity ${ }^{6,13-16}$, while the ability of the fibrils to "shed" oligomer and protofibril species may also contribute to cellular toxicity and propagation ${ }^{17,18}$. Previous studies have identified some of the cellular-level details of the internalization, seeding, and propagation of $\alpha S$ fibrils $^{10,11,19-27}$. However, these studies lack the information needed to understand the molecular details of how fibrils can template further aggregation, and critically, the mechanisms by which $\alpha S$ fibril seeding of endogenous $\alpha \mathrm{S}$ is affected by inhibitors of $\alpha \mathrm{S}$ aggregation.

Beta-synuclein ( $\beta S$ ), a homologous protein which is co-localized with $\alpha \mathrm{S}$ and is expressed at variable levels relative to $\alpha S$ in different synucleinopathies ${ }^{28,29}$, has been recognized as a natural inhibitor of $\alpha S$ aggregation ${ }^{30}$. A transgenic mouse model that simultaneously expresses both human $\alpha \mathrm{S}$ and $\beta S$ had fewer inclusions and less neurodegeneration compared with only $\alpha S$-expressing transgenic mice ${ }^{30}$. Interestingly, no detectable amount of $\beta S$ has been found in $\mathrm{LB}^{31,32}$ even though $\beta S$ can be over-expressed in certain parts of the PD brain ${ }^{28}$, begging the question of how exactly $\beta S$ interacts with $\alpha S$ to provide neuroprotection and influence $\alpha S$ fibril-induced cellular toxicity.

We have previously investigated the sequence and domain level interactions that mediate the influence of $\beta S$ on the aggregation and fibril formation of $\alpha S^{33,34}$. We have found that head-to-tail transient complexes between $\beta S$ and $\alpha S^{35}$, mediated by multi-pronged $\mathrm{N}$ - and $\mathrm{C}$-terminal interactions ${ }^{33}$, provide enough of a kinetic trap at the earliest stages of $\alpha S$ aggregation to slow down the assembly of $\alpha S$ into fibrils. However, even though $\beta S$ slows down $\alpha S$ aggregation and reduces the overall $\alpha S$ fibril load in a concentration dependent manner ${ }^{35,36}$, it does not

\footnotetext{
${ }^{1}$ Department of Chemistry and Chemical Biology, Rutgers University, Piscataway, New Jersey, 08854, USA. ${ }^{2}$ RWJMS Institute for Neurological Therapeutics, Rutgers Biomedical and Health Sciences, and Department of Neurology, Robert Wood Johnson Medical School, Rutgers University, Piscataway, New Jersey, 08854, USA. ${ }^{3}$ These authors contributed equally: Xue Yang and Jonathan K.Williams. *email: baum@chem.rutgers.edu
} 

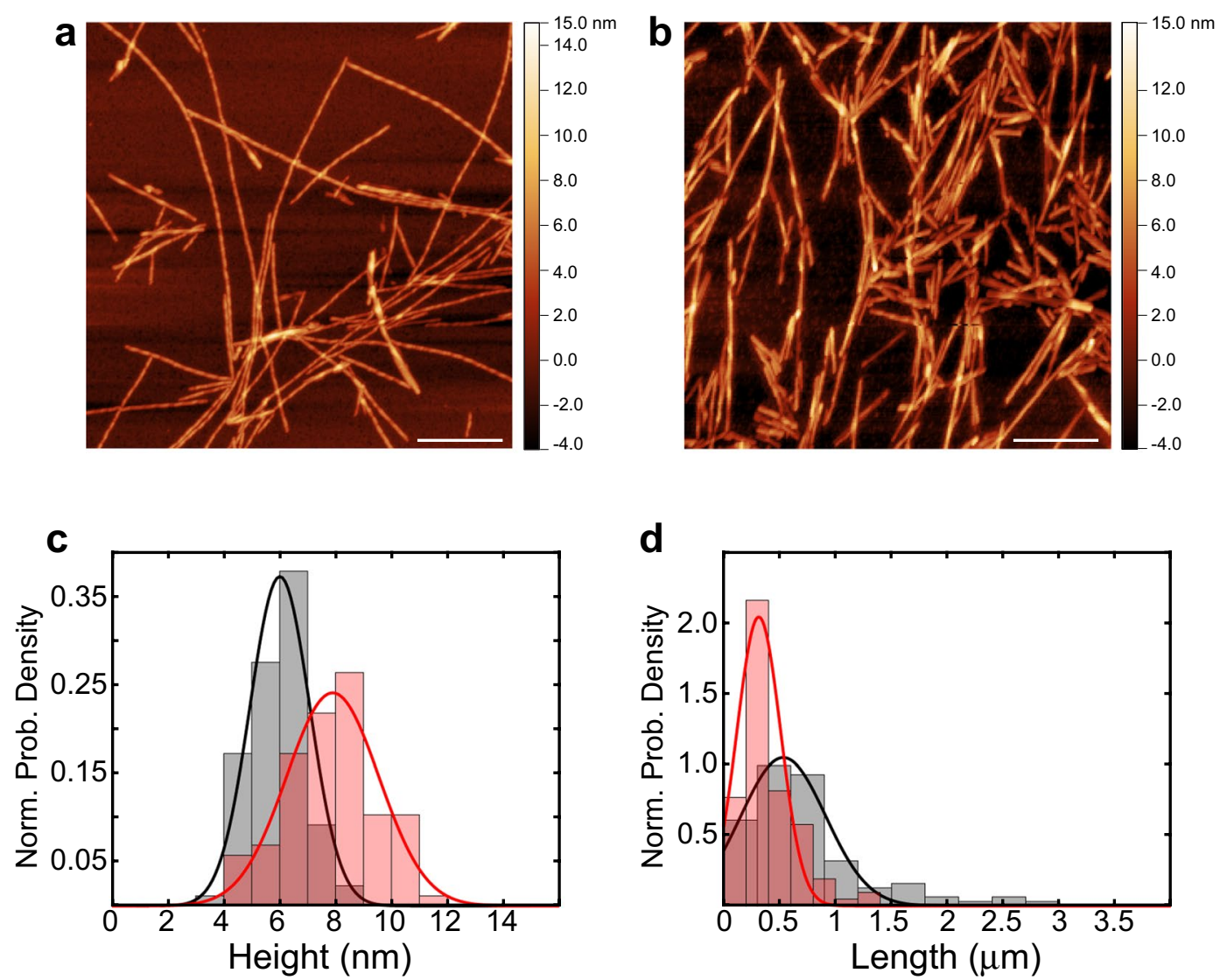

Figure 1. Morphological differences between $\alpha S$ and co-incubated $\alpha S / \beta S$ fibrils. (a,b) Amplitude modulated (AM) AFM images of fibrils formed from (a) $70 \mu \mathrm{M}$ monomeric $\alpha \mathrm{S}$ and (b) $70 \mu \mathrm{M}$ monomeric $\alpha \mathrm{S}$ co-incubated with $210 \mu \mathrm{M}$ monomeric $\beta S$. The $\mathrm{x}-\mathrm{y}$ axes length scale bar is $500 \mathrm{~nm}$. Assessment of the height (c) and length (d) of $\alpha S$ (black) versus co-incubated $\alpha S / \beta S$ (red) fibrils. Histograms of height and length data are presented as normalized probability densities, and the best-fit probability density function is overlaid to better visualize the distributions.

fully abolish $\alpha \mathrm{S}$ fibril formation. Therefore, a detailed understanding of the conformational properties and cytotoxicity of $\alpha S$ fibrils formed in the presence of $\beta S$ will provide us with a deeper understanding of the mechanisms underlying fibril toxicity.

Here, we show that co-incubation of the monomeric intrinsically disordered $\alpha S$ with the monomeric intrinsically disordered $\beta S$ results in unique "co-incubated $\alpha S / \beta S$ " fibrils that show a significant reduction in cellular toxicity, a reduction in seeding capacity, and are more resistant to fibril shedding. Solid-state NMR experiments revealed that while the overall structure of the core of $\alpha S / \beta S$ fibrils is minimally perturbed, the imperfect KTKEGV consensus motif repeats of $\alpha \mathrm{S}$ in the preNAC N-terminus become dynamic and more water accessible. Our results offer insight into the mechanism of amyloid fibril toxicity and highlight that increased dynamics of co-incubated $\alpha \mathrm{S} / \beta \mathrm{S}$ fibrils may interfere with their templating ability, thereby reducing their seeding capacity. Targeting amyloid fibrils by enhancing their dynamics may be a new strategy in designing therapeutics against neurodegenerative diseases.

\section{Results}

Co-incubation with $\beta S$ induces subtle differences in $\alpha S$ fibril morphology. We studied the differences in the morphology of $\alpha S$ fibrils formed from the incubation of monomeric N-terminally acetylated $\alpha S$, and $\alpha \mathrm{S}$ fibrils formed by co-incubation of monomeric $\mathrm{N}$-terminally acetylated $\alpha \mathrm{S}$ with monomeric $\mathrm{N}$-terminally acetylated $\beta S$ at a 1:3 ratio (called $\alpha \mathrm{S} / \beta \mathrm{S}$ co-incubated fibrils). We have used the $\mathrm{N}$-terminally acetylated forms of $\alpha S$ and $\beta S$, since this post-translational modification is constitutively present in the native forms of these intrinsically disordered proteins ${ }^{37,38}$. Consistent with our previous work ${ }^{33,35}, \beta S$ delays $\alpha S$ fibril formation in the Thioflavin T (ThT) aggregation assay (Fig. S1), whereby the co-incubation of $\alpha S$ with $\beta S$ results in a longer lag time and slower growth kinetics compared with $\alpha \mathrm{S}$ by itself. Fibrils formed as the end products of these two monomer aggregation assays display differences in their polymorph composition. Atomic force microscopy (AFM) images show that $\alpha \mathrm{S}$ by itself (Fig. 1a) forms long straight or twisted fibril polymorphs, similar to previous reports $^{13,39,40}$, while co-incubated $\alpha S / \beta S$ forms fibril polymorphs that are shorter and straight (Fig. $1 b$ ), with no discernable twisting pattern. On average, the height of $\alpha S$ fibrils $(6.0 \pm 1.1 \mathrm{~nm})$ tends to be shorter than $\alpha \mathrm{S} / \beta \mathrm{S}$ 


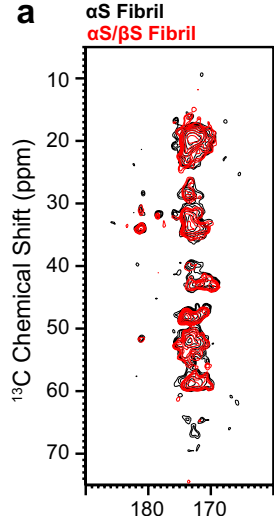

b as Fibril

b as Fibril

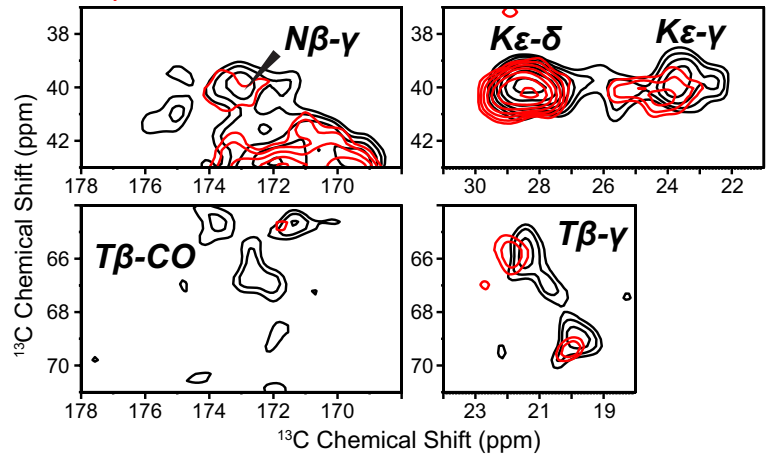

C as Fibril

C $\quad{ }_{2 \mathrm{NOA}}^{\alpha S \mathrm{~F}}$

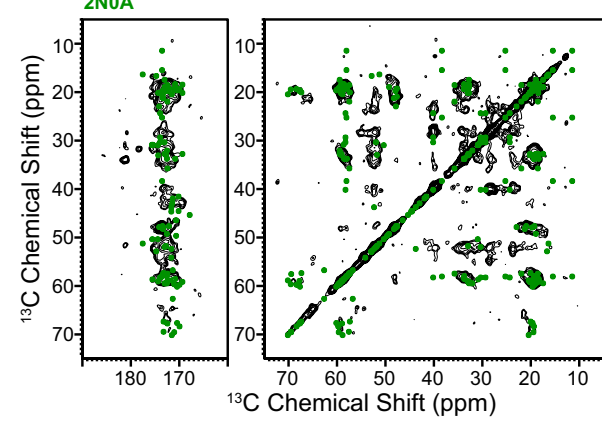

d as Fibril

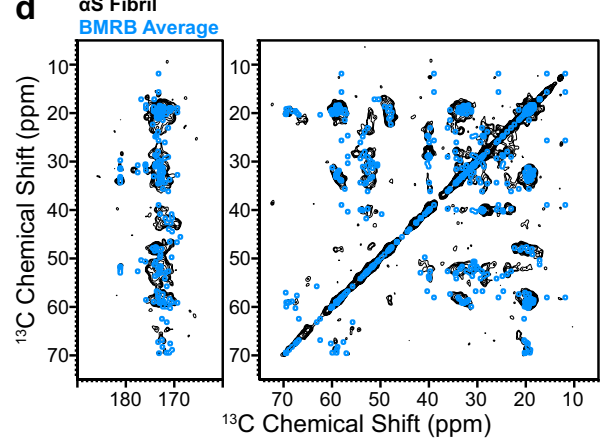

Figure 2. Characterization of the Fibril Core. (a) Overlay of $\alpha S$ fibril (black) and co-incubated $\alpha S / \beta S$ fibril (red) $2 \mathrm{D}^{13} \mathrm{C}-{ }^{13} \mathrm{C}$ correlation spectra (100 ms DARR), showing that the conformation of the core does not differ significantly between these two fibrils. (b) Expansion of select regions of the $2 \mathrm{D}{ }^{13} \mathrm{C}-{ }^{13} \mathrm{C}$ spectra in (a) that show the major cross peak intensity differences, which are the Lys, Thr and a tentatively assigned Asn residues. (c,d) Overlays of the $\alpha S 2 D^{13} \mathrm{C}^{13} \mathrm{C}$ correlation spectrum in (a) with chemical shift correlation maps derived from (c) the published solid-state NMR structure of $\alpha$ S fibrils (green, PDB: 2N0A, BMRB: $25518^{41}$ ) and (d) an average of the ${ }^{13} \mathrm{C}$ chemical shifts of $\alpha$ S fibrils deposited into the BMRB (BRMB: $16939^{43}, 17498^{44}, 17910^{45}, 18243^{46}$, $\left.18860^{44}, 25518^{41}, 25535^{47}, 26890^{48}\right)$.

fibrils (7.9 $\pm 1.7 \mathrm{~nm})$ (Fig. 1c), while the length of $\alpha \mathrm{S} / \beta S$ fibrils $(0.3 \pm 0.2 \mu \mathrm{m})$ tend to be shorter than $\alpha \mathrm{S}$ fibrils $(0.5 \pm 0.4 \mu \mathrm{m})$ (Fig. $1 \mathrm{~d})$.

We quantified the monomer composition of the co-incubated $\alpha \mathrm{S} / \beta \mathrm{S}$ fibrils to try to ascertain whether these morphological changes could be induced by incorporation of $\beta S$ into the protofilaments that make up the fibril. Mature fibrils were solubilized in $4 \mathrm{M}$ guanidine hydrochloride and analyzed by ESI-MS. Surprisingly, the co-incubated $\alpha \mathrm{S} / \beta \mathrm{S}$ fibrils are composed of less than $6 \% \beta S$ (Fig. S1).

$\alpha S / \beta S$ co-incubated fibril core structure is maintained while the $\mathrm{N}$-terminal dynamics are increased. We investigated the conformational and dynamics properties of the $\alpha \mathrm{S}$ fibril when co-incubated with $\beta$ S utilizing solid-state NMR (ssNMR) spectroscopy. We first assessed the secondary structure of our fibrils from $2 \mathrm{D}{ }^{13} \mathrm{C}-{ }^{13} \mathrm{C}$ and ${ }^{15} \mathrm{~N}-{ }^{13} \mathrm{C}$ correlation spectra. Figure 2 a shows the $2 \mathrm{D}{ }^{13} \mathrm{C}-{ }^{13} \mathrm{C} 100 \mathrm{~ms}$ DARR spectra and Fig. S5 the $2 \mathrm{D}^{15} \mathrm{~N}^{-13} \mathrm{C}$ correlation spectra of $\alpha \mathrm{S}$ fibrils in black overlaid with co-incubated $\alpha \mathrm{S} / \beta \mathrm{S}$ fibrils in red. This experiment utilizes a cross-polarization period to transfer ${ }^{1} \mathrm{H}$ to ${ }^{13} \mathrm{C}$ magnetization, which preferentially detects the rigid residues that make up the core of the fibril and does not detect the dynamic or disordered residues that make up the bulk of the $\mathrm{N}$ - and $\mathrm{C}$-terminal regions. The ${ }^{13} \mathrm{C}$ chemical shifts, ${ }^{13} \mathrm{C}-{ }^{13} \mathrm{C}$ cross peaks, and ${ }^{15} \mathrm{~N}-{ }^{13} \mathrm{C}$ cross peaks of the spectra do not show marked differences between $\alpha \mathrm{S}$ and $\alpha \mathrm{S} / \beta \mathrm{S}$ fibrils, indicating that the core structure of the fibril does not change even when formed in the presence of a stoichiometric excess of $\beta S$ (Fig. 2a). To ascertain whether our fibrils maintain the common Greek-key motif core structure previously identified $^{13,39-41}$, without conducting a full structure determination by ssNMR, we compared our spectra with the ${ }^{13} \mathrm{C}$ chemicals shifts of $\alpha \mathrm{S}$ fibrils deposited into the Biological Magnetic Resonance Data Bank ${ }^{42}$ (BMRB). Out of 15 total entries in the BMRB of $\alpha$ S fibril chemical shifts ( 8 human WT, 6 human mutants, 1 mouse WT) only one is associated with a high-resolution 3D structural model of human WT $\alpha$ S fibrils (BMRB 25518, PDB 2N0A) ${ }^{41}$. Using the ${ }^{13} \mathrm{C}$ chemical shifts from BMRB 25518 we created a ${ }^{13} \mathrm{C}-{ }^{13} \mathrm{C}$ chemical shift correlation map, shown in green in Fig. $2 c$, and overlaid it with our $\alpha \mathrm{S}$ fibril ${ }^{13} \mathrm{C}-{ }^{13} \mathrm{C}$ spectrum; we observe relatively good agreement between the published chemical shifts and our $\alpha \mathrm{S}$ spectrum. In addition, we created $\mathrm{a}^{13} \mathrm{C}-{ }^{13} \mathrm{C}$ chemical shift correlation map using an average of the ${ }^{13} \mathrm{C}$ chemical shifts from the core residues (44-96) in the 8 human WT entries in the BMRB (Entries: $16939^{43}, 17498^{44}, 17910^{45}, 18243^{46}, 18860^{44}, 25518^{41}, 25535^{47}, 26890^{48}$ ) (Fig. 2d). 
When overlaid with our $\alpha S$ fibril spectrum, we again find relatively good agreement between the two spectra. This comparison between the previously published ${ }^{13} \mathrm{C}$ chemical shifts, in particular the ${ }^{13} \mathrm{C}$ chemical shifts from $\mathrm{PDB} 2 \mathrm{~N} 0 \mathrm{~A}$, and our own ${ }^{13} \mathrm{C}$ spectra suggests that the secondary structure of our $\alpha \mathrm{S}$ fibril core is consistent with a Greek-key topology. A full structure determination and peak assignment is currently underway to confirm this assessment.

While the core of our $\alpha S$ and co-incubated $\alpha S / \beta S$ fibrils are unchanged, there are some subtle differences in peak intensities between $\alpha \mathrm{S}$ and $\alpha \mathrm{S} / \beta \mathrm{S}$ fibrils. The $\mathrm{C} \beta-\mathrm{C} \alpha, \mathrm{C} \beta-\mathrm{C} \gamma$ and $\mathrm{C} \beta-\mathrm{CO}$ cross-peaks of the threonine residues show marked intensity decreases in the co-incubated fibril (Fig. 2b). We also observed intensity decreases in the cross peaks of lysine $\mathrm{C} \varepsilon-\gamma$ and $\mathrm{C} \beta-\gamma$ of N65 of the co-incubated fibril (Fig. 2b); the N65 C $\beta-\gamma$ assignment is tentative, and could also plausibly be from I88 C $\beta$-CO or F94 C $\beta$-CO. The loss of intensity of these peaks could be caused by increased dynamics of these residues in the co-incubated fibril. To further investigate this point we measured the ${ }^{13} \mathrm{C} \mathrm{T}_{1 \rho}$ relaxation time ${ }^{49}$, which reports on $\mu$ s timescale dynamics, of the threonine $\mathrm{C} \beta$, since these peaks are well resolved from chemical shift overlap of any other residue in the region from $\sim 65-70 \mathrm{ppm}$ (Fig. $2 \mathrm{a}$ and Fig. S4a,b). We found that the ${ }^{13} \mathrm{C} \mathrm{T}_{10}$ relaxation time of the $\mathrm{Thr} C \beta$ 's from co-incubated $\alpha \mathrm{S} / \beta S$ fibrils decreased relative to the Thr C $\beta$ 's from $\alpha$ S fibrils (Fig. S4c), indicating an increase in dynamics of these residues.

To further characterize any differences between $\alpha S$ and co-incubated $\alpha S / \beta S$ fibrils, we probed the changes in water accessibility and hydration between the two fibrils. Figure 3 shows the water-edited $2 \mathrm{D}{ }^{13} \mathrm{C}-{ }^{13} \mathrm{C}$ correlation spectra ${ }^{50,51}$ of ${ }^{13} \mathrm{C},{ }^{15} \mathrm{~N}$-labeled $\alpha S$ fibrils (Fig. 3a) and co-incubated $\alpha \mathrm{S} / \beta \mathrm{S}$ fibrils where only $\alpha \mathrm{S}$ is uniformly labeled with ${ }^{13} \mathrm{C}$ and ${ }^{15} \mathrm{~N}$ (Fig. 3b). The basic premise of this experiment is to observe how the transfer of water ${ }^{1} \mathrm{H}$ magnetization varies across the fibril. The long water ${ }^{1} \mathrm{H}$ spin-diffusion (SD) mixing time (100 ms, black) spectra represent a state where the water ${ }^{1} \mathrm{H}$ magnetization has fully equilibrated across each fibril, while the short water ${ }^{1} \mathrm{H}$ SD mixing time ( $3 \mathrm{~ms}$, red) spectra illustrate the fibril residues that are in closest proximity to water. The relative proximity or accessibility of a residue to water is then most easily compared by taking the ratio between these two intensities $\left(\mathrm{Int}_{3 \mathrm{~ms}} / \mathrm{Int}_{100 \mathrm{~ms}}\right.$ ). Due to sample sensitivity and time constraints, in lieu of obtaining residue specific full SD build up curves, the reason for using the ratio between the short and long mixing times is to provide normalization of the intensities measured in the short mixing time experiment and allow for a relative comparison of the initial spin-diffusion buildup rates between different samples. For example, residues that are far from water or are located in the center of the fibril core will have smaller water-accessibility ratios, while residues that are on the surface of the fibril will have larger water-accessibility ratios. This approach has been used previously to probe the differential hydration environments of amyloid fibrils and other biomolecules ${ }^{51-55}$.

Slices from the water edited $2 \mathrm{D}^{13} \mathrm{C}-{ }^{13} \mathrm{C}$ spectra show decreases in the relative intensities of several of the threonine and lysine cross-peaks of the $\alpha S / \beta S$ fibrils compared to $\alpha S$ fibrils, while those lysine cross-peaks that remain in the $\alpha S / \beta S$ fibril spectra have increased water spin-diffusion (i.e. larger $\operatorname{Int}_{3 \mathrm{~ms}} / \mathrm{Int}_{100 \mathrm{~ms}}$ ratios) relative to $\alpha S$ fibrils (Fig. 3c). The increase in water spin-diffusion of the $\alpha S / \beta S$ fibril lysine peaks indicates that these residues are more water accessible. Conversely, the water spin diffusion ratios of the hydrophobic alanine and valine residues do not change between $\alpha S$ and co-incubated $\alpha S / \beta S$ fibrils (Fig. 3c), indicating that the hydration environment of these residues does not significantly change between the two fibrils.

Co-incubated fibrils are more sensitive to proteinase $\mathrm{K}$ digestion. Proteasomal impairment has been implicated in several neurodegenerative diseases ${ }^{56}$, including PD $^{57}$, and as proteasome activity decreases with age cells become more vulnerable to deleterious protein aggregation ${ }^{58}$. Therefore, an understanding of how synuclein fibrils and aggregates undergo protease degradation and clearance may shed critical light on PD progression. In order to understand the differences in protease degradation and fibril stability between $\alpha \mathrm{S}$ fibrils and co-incubated $\alpha S / \beta S$ fibrils, we carried out a series of digestion assays with increasing concentrations of proteinase $\mathrm{K}$ (Fig. 3c). We observed that co-incubated $\alpha S / \beta S$ fibrils are more sensitive to proteinase $\mathrm{K}$ digestion at a concentration of $5 \mu \mathrm{g} / \mathrm{ml}$. The co-incubated fibrils display an enhanced propensity to be degraded to low molecular weight species (i.e. intense band at $\sim 5 \mathrm{kDa}$ only) compared to $\alpha \mathrm{S}$ fibrils, which have a larger proportion of high molecular species (i.e. intense bands at $\sim 10$ and $\sim 15 \mathrm{kDa}$ ).

Co-incubated $\alpha S / \beta S$ fibrils are less toxic and exhibit reduced seeding and proliferation capacity compared to $\alpha S$ fibrils in neuroblastoma cells. Having now characterized the conformational and polymorphic differences between $\alpha S$ and co-incubated $\alpha S / \beta S$ fibrils, we investigated how these changes in protofilament packing and dynamics affects the cytotoxicity of the fibrils. It is common practice to sonicate amyloid fibrils directly before use in cell toxicity assays, in order to obtain a more uniform distribution of fibril lengths and size. However the sonication process also produces smaller aggregates and oligomers ${ }^{59}$, with an unknown distribution of shapes and sizes, that show different toxicities, fibril seeding abilities, and cell incorporation capacities ${ }^{19}$. In order to avoid forming these additional oligomers, we have elected to use un-sonicated fibrils in our assays of cell toxicity and seeding, with polymorph compositions (Fig. 1a,b) and size distributions (Fig. 1c,d) illustrated in our AFM images. As a consequence of using long un-sonicated fibrils, the concentration of fibrils used in our assays $(\sim 1 \mu \mathrm{M})$ is higher than the $\mathrm{nM}$ concentrations used previously for smaller sonicated fibrils, since these smaller sonicated species are more easily incorporated into cells ${ }^{60,61}$. However, our fibril preparations are internalized similarly to sonicated fibrils, showing the characteristic fluorescent punctate-like structures (Fig. S7).

Fibrils of $\alpha S$ or $\alpha S / \beta S$ were added to cultures of human SH-SY5Y neuroblastoma cells and incubated for 24 hours, at which time cellular viability was assessed by the ability of the cells to reduce 3-(4, 5-dimethylthiazol-2 -yl)-5-(3-carboxymethoxyphenol)-2-(4-sulfophenyl)-2H-tetrazolium (MTS). Compared with untreated cells, or cells treated with monomeric $\alpha S$, fibrils of $\alpha \mathrm{S}$ induced a $30 \%$ reduction in cell viability $(* * * \mathrm{p}<0.001)$, whereas $\alpha \mathrm{S} / \beta \mathrm{S}$ fibrils had no significant impact on cell viability (Fig. 4a).

We also investigated the ability of $\alpha S$ and $\alpha S / \beta S$ fibrils to seed aggregation in vitro using a quiescent thioflavin-T (ThT) fluorescence assay. Figure S3 shows that while preformed seeds of both $\alpha \mathrm{S}$ and $\alpha \mathrm{S} / \beta \mathrm{S}$ fibrils 

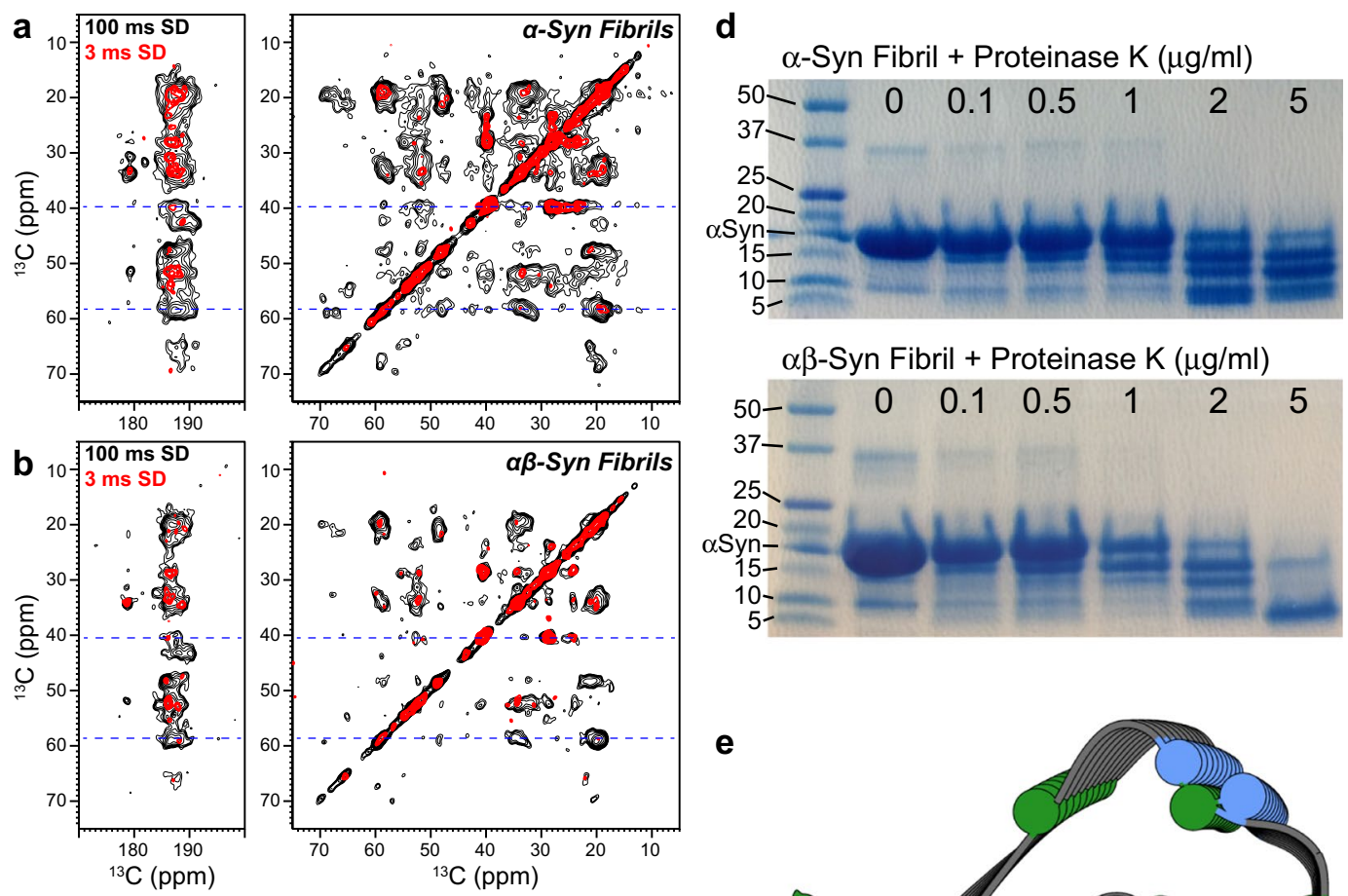

C
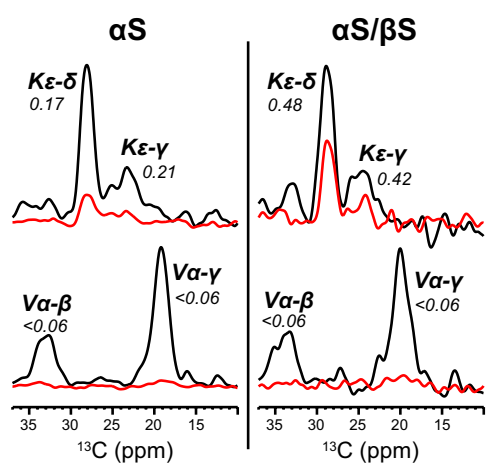

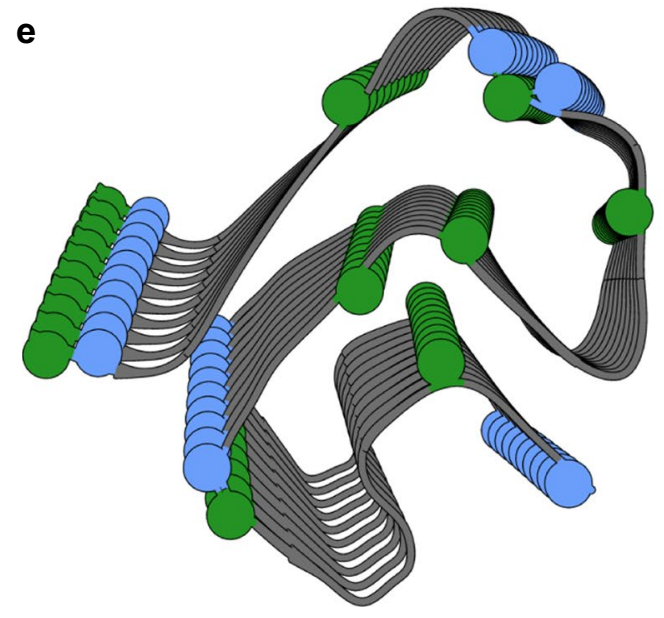

Figure 3. Changes in fibril water accessibility and fibril degradation. (a-c) Water-edited solid-state NMR ${ }^{13} \mathrm{C}-$ ${ }^{13} \mathrm{C}$ correlation spectra of (a) $\alpha \mathrm{S}$ and (b) co-incubated $\alpha S / \beta S$ fibrils. Magnetization was equilibrated at long water spin-diffusion times $(100 \mathrm{~ms}$, black) compared with the initial water-protein magnetization transfer at short spin-diffusion times $(3 \mathrm{~ms}$, red). (c) 1D slices taken at the blue dashed lines in (a) and (b) of $\alpha S$ fibrils (left side) and $\alpha \mathrm{S} / \beta \mathrm{S}$ fibrils (right side), showing the intensities of cross-peaks to lysine (top) or valine (bottom) side chains. The ratio of the cross-peak intensities at long and short spin diffusion times $\left(\mathrm{Int}_{3 \mathrm{~ms}} / \mathrm{Int}_{100 \mathrm{~ms}}\right)$ indicates the relative proximity of water on a residue-specific basis. (d) Digestion of $\alpha S$ and $\alpha S / \beta S$ fibrils at various concentrations of proteinase K. Full-length gels are presented in Supplementary Fig. 6. (e) Map of the residues that show the largest degree of change in water accessibility between $\alpha \mathrm{S}$ and $\alpha \mathrm{S} / \beta \mathrm{S}$ fibrils, lysine (blue) and threonine (green), on the core-residues (44-96) of PDB structure 2N0A.

have the ability to induce fibril formation in the presence of $\alpha S$ monomers, the time for the ThT fluorescence curve to plateau takes longer with $\alpha S / \beta S$ fibril seeds. This indicates that $\alpha S / \beta S$ fibrils have a reduced capacity to seed further $\alpha S$ aggregation. We confirmed these observations of $\alpha S$ and $\alpha S / \beta S$ fibril seeding capacity in cell, by assessing the ability of these fibrils to seed aggregation of endogenous $\alpha \mathrm{S}$ in SH-SY5Y cells through the analysis of the fluorescence intensities of dyes that specifically bind to $\alpha S$ and amyloid structures (Fig. 4b). Cells were treated with monomeric $\alpha S$, $\alpha S$ fibrils or $\alpha S / \beta S$ fibrils for 24 hours before being fixed and stained with purified mouse anti- $\alpha S$ (anti- $\alpha$-synuclein) antibody, thioflavin S (ThioS), and 4',6-diamidino-2-phenylindole (DAPI). Cells were then imaged by confocal fluorescence microscopy, where the anti- $\alpha \mathrm{S}$ antibody fluoresces red and indicates the presence of any synuclein species present, ThioS fluoresces green and indicates the formation of amyloid species, and DAPI stains the cell nucleus blue (Fig. 4b). Compared with cells treated with monomeric $\alpha S$ (Fig. 4b, bottom row), cells treated with $\alpha S$ fibrils showed an increase in anti- $\alpha S$ antibody fluorescence of $7.3 \times$ (Fig. $4 \mathrm{~b}$, top row), while cells treated with $\alpha \mathrm{S} / \beta \mathrm{S}$ fibrils showed a smaller increase of $4.4 \times$ (Fig. $4 \mathrm{~b}$, middle row). ThioS staining indicating amyloid formation showed a similar trend with a $4.8 \mathrm{x}$ increase with $\alpha$ S fibrils vs a $3.4 \mathrm{x}$ increase with $\alpha \mathrm{S} / \beta \mathrm{S}$ fibrils. 


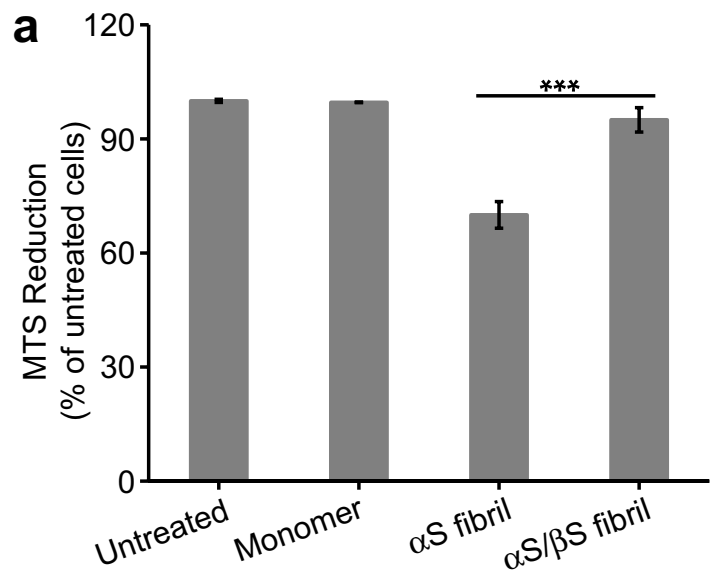

b Anti- $\alpha-S y n c u l e i n$

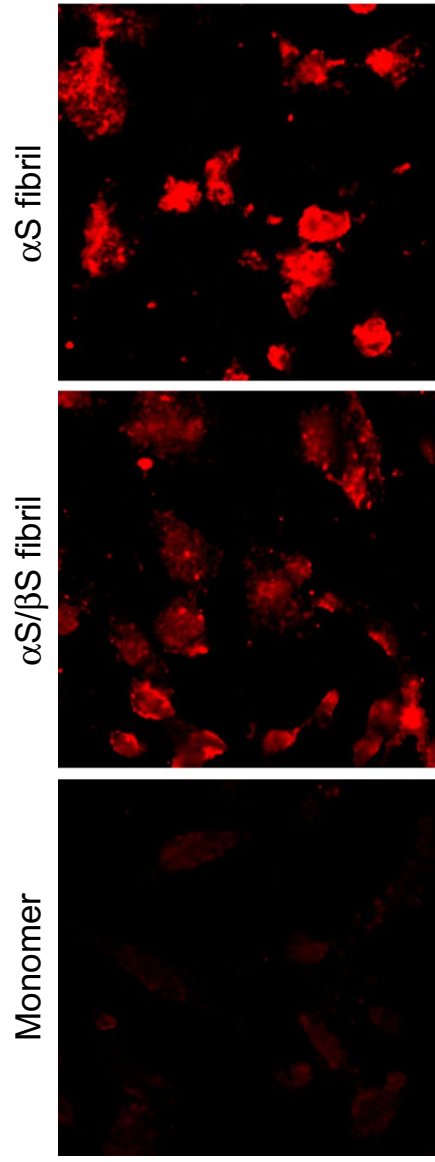

Thios
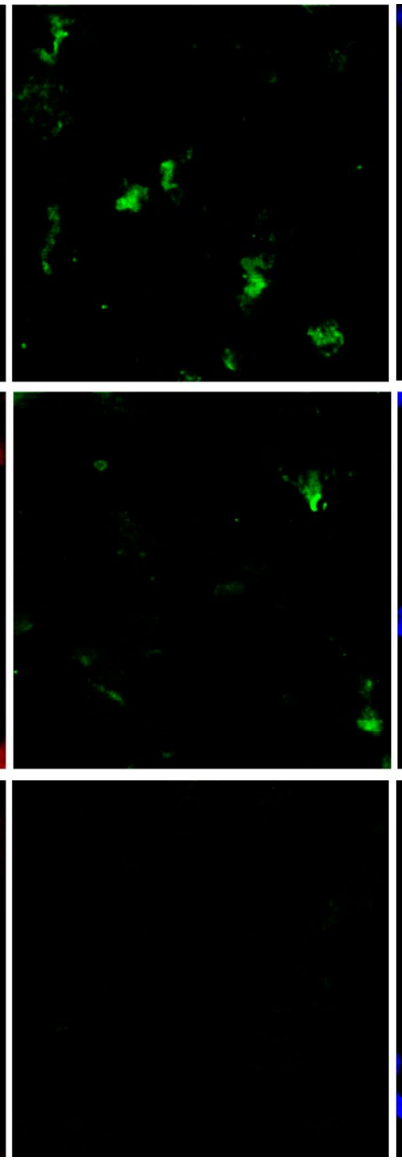

DAPI
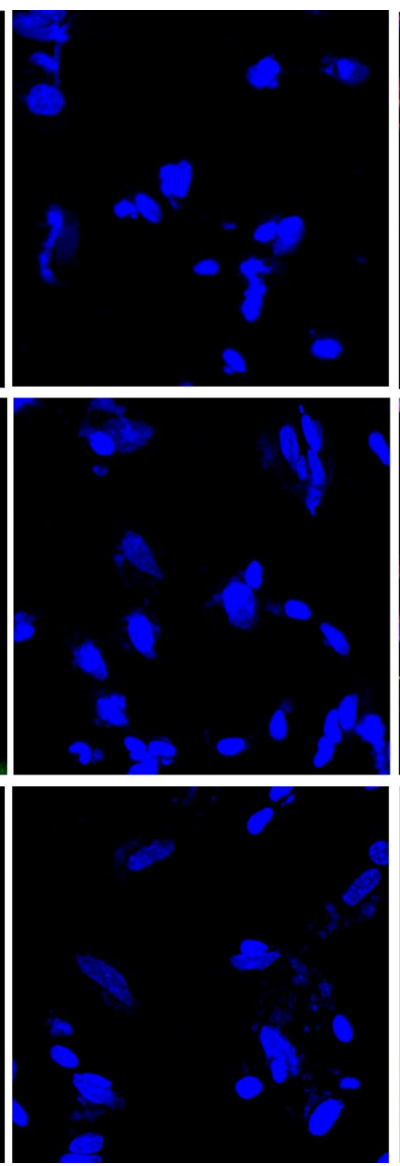

Merge
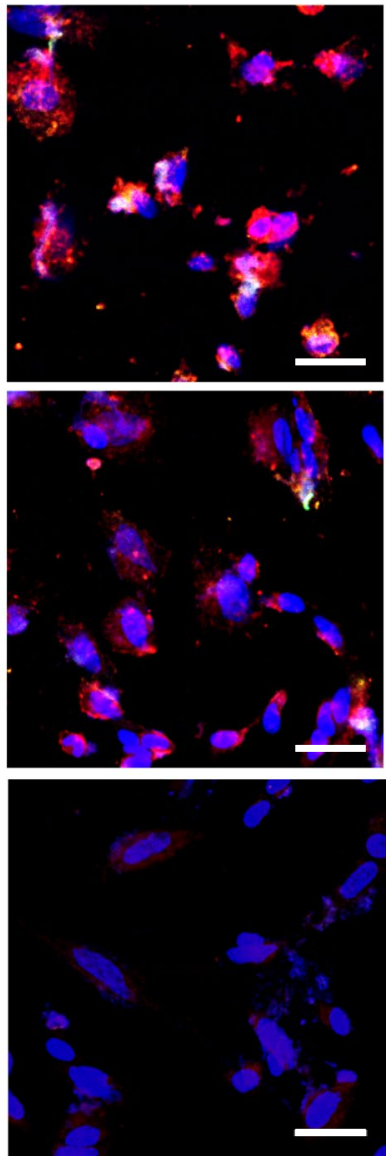

Figure 4. Cellular toxicity of $\alpha S$ and co-incubated $\alpha S / \beta S$ fibrils and their seeding potential. (a) Viability of SHSY5Y cells assessed by MTS assay after treatment with $\alpha \mathrm{S}$ or $\alpha \mathrm{S} / \beta \mathrm{S}$ fibrils ( $1.3 \mu \mathrm{M}$ monomer equivalents), or monomeric $\alpha \mathrm{S}(1.3 \mu \mathrm{M})$ as control, for 24 hours. Data shown are means and standard errors of the mean (SEM) of 3 independent experiments run in triplicates. ***ANOVA $\mathrm{p}<0.001$. (b) Confocal fluorescence microscopy images of SH-SY5Y cells treated with $1.3 \mu \mathrm{M} \alpha \mathrm{S}$ fibril (top), $\alpha \mathrm{S} / \beta \mathrm{S}$ fibril (middle), or $\alpha \mathrm{S}$ monomer (bottom) for 24 hours before fixing and staining. Separate channels are presented showing the presence of all $\alpha \mathrm{S}$ species (anti- $\alpha$ S antibody, red), all amyloid species (ThioS, green), and cell nuclei (DAPI, blue), along with the three channels overlaid (merge). The scale bar is $40 \mu \mathrm{m}$.

Oligomers shed from $\alpha S$ or $\alpha S / \beta S$ fibrils have different morphologies, toxicities and seeding capacities. It has been hypothesized that as the endpoint of misfolding and aggregation of several neurodegenerative disease associated proteins, amyloid fibrils might act as a "sink" to sequester misfolded toxic species ${ }^{62}$. However, amyloid fibrils do not represent a completely stable species in solution, rather they exist in a dynamic equilibrium between fibril and oligomer forms. Indeed, toxic oligomers have even been observed to shed from 
a

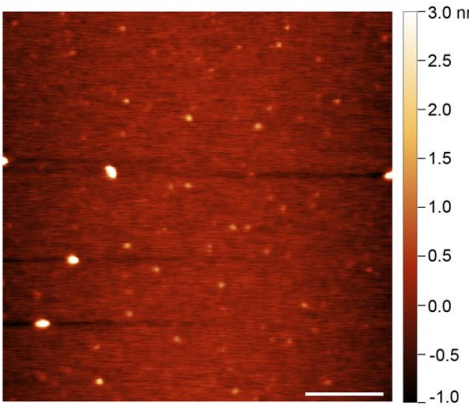

b

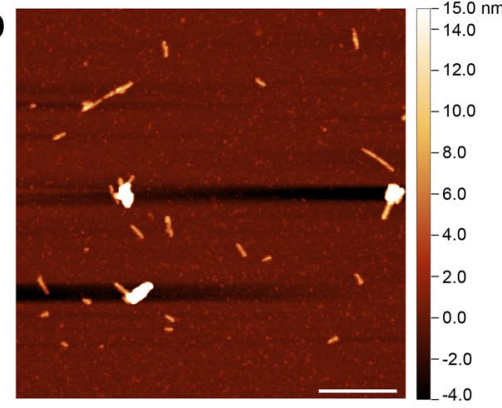

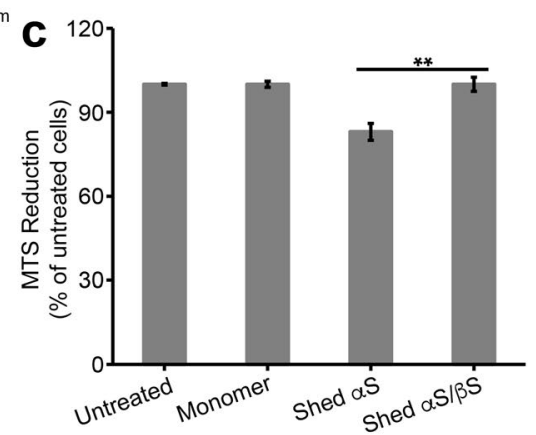

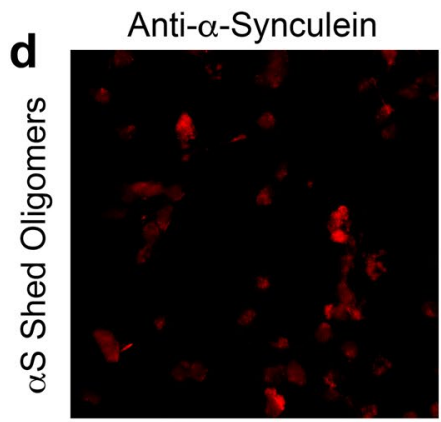

Thios

DAPI
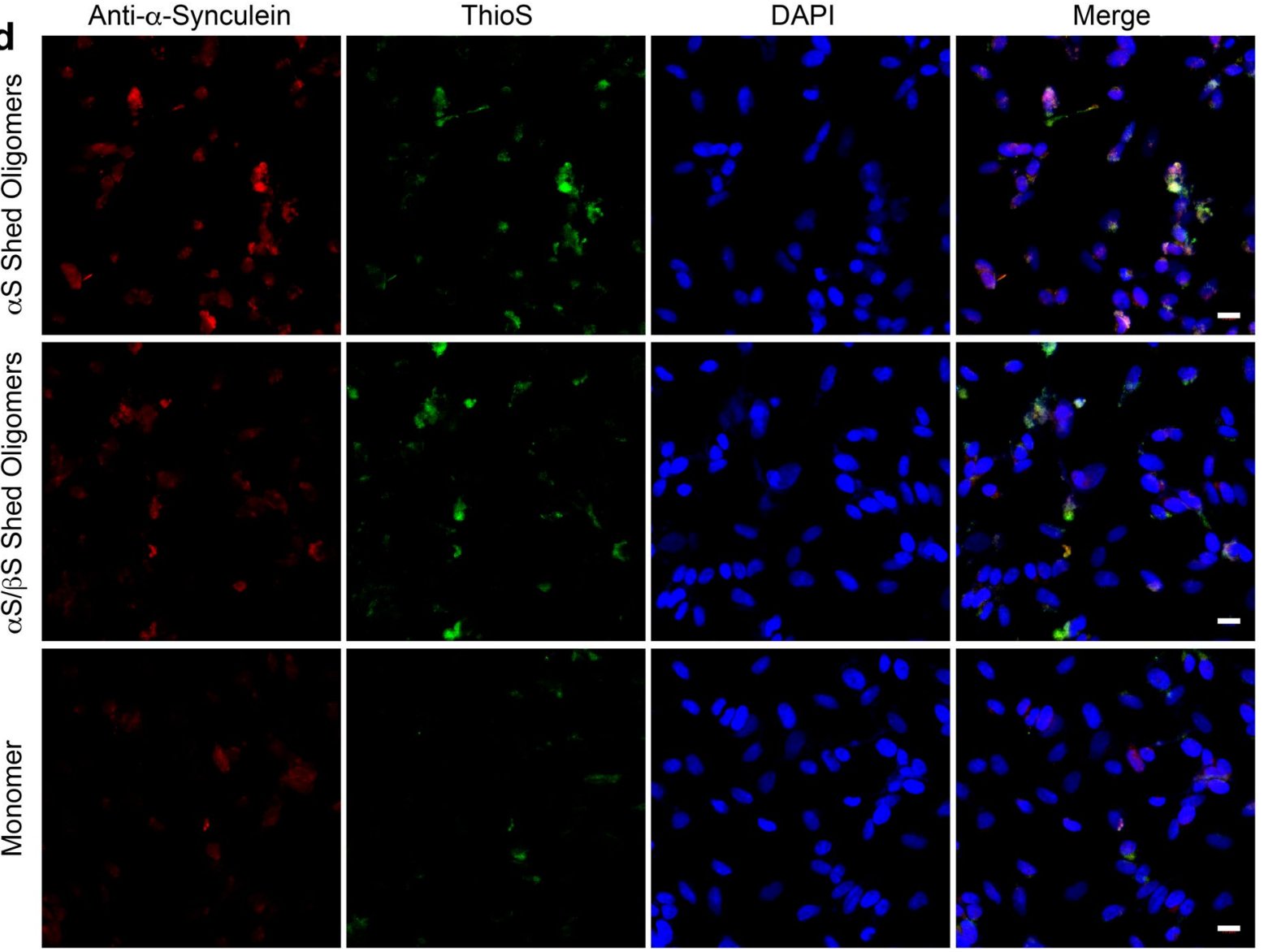

Figure 5. Morphology and toxicity of oligomeric species that are shed from mature fibrils. (a,b) AM-AFM images of the oligomeric species that are shed from mature $\alpha \mathrm{S}$ fibrils $(\mathbf{a})$ and mature $\alpha S / \beta S$ fibrils $(\mathbf{b})$. The length scale bar is $500 \mathrm{~nm}$. (c) Viability of SH-SY5Y cells assessed by MTS assay after treatment for 48 hours with the shed oligomers from $\alpha$ S fibrils or shed oligomers from $\alpha \mathrm{S} / \beta S$ fibrils $(0.7 \mu \mathrm{M}$ monomer equivalents), or with monomeric $\alpha S(0.7 \mu \mathrm{M})$ as a control. Data shown are means \pm SEM of 3 independent experiments run in triplicates. **ANOVA p $<0.01$. (d) Confocal fluorescence microscopy images of SH-SY5Y cells treated with oligomers shed from $\alpha S$ fibrils (top), oligomers shed from $\alpha S / \beta S$ fibrils (middle), or with $\alpha S$ monomer (bottom) for 48 hours before fixing and staining. Separate channels are presented showing the presence of all $\alpha \mathrm{S}$ species (anti- $\alpha$ S antibody, red), all amyloid species (ThioS, green), and cell nuclei (DAPI, blue), along with the three channels overlaid (merge). The scale bar is $16 \mu \mathrm{m}$.

mature $\alpha S$ fibrils over time ${ }^{18}$. To understand the effect of $\beta S$ on the stability and equilibrium of $\alpha S$ fibrils, we sought to determine the morphology, toxicity and cell seeding capacities of the oligomers that are shed from $\alpha S$ fibrils and $\alpha S / \beta S$ fibrils.

We first measured the thermostability of the two fibrils using far-UV circular dichroism (CD) spectroscopy. The CD spectra show that both $\alpha \mathrm{S}$ and $\alpha \mathrm{S} / \beta \mathrm{S}$ fibrils have the characteristic spectral minimum at $218 \mathrm{~nm}$, indicating the presence of $\beta$-sheet structure (Fig. S2). We monitored the change in ellipticity of the $218 \mathrm{~nm}$ signal as a function of temperature, and found that change in ellipticity of co-incubated $\alpha S / \beta S$ fibrils is less than that of $\alpha S$ fibrils as temperature increased, indicating that $\alpha S / \beta S$ fibrils are more thermostable than $\alpha S$ fibrils (Fig. S2). AFM images show that the oligomers that are shed from $\alpha$ S fibrils (Fig. 5a) primarily adopt small globular 


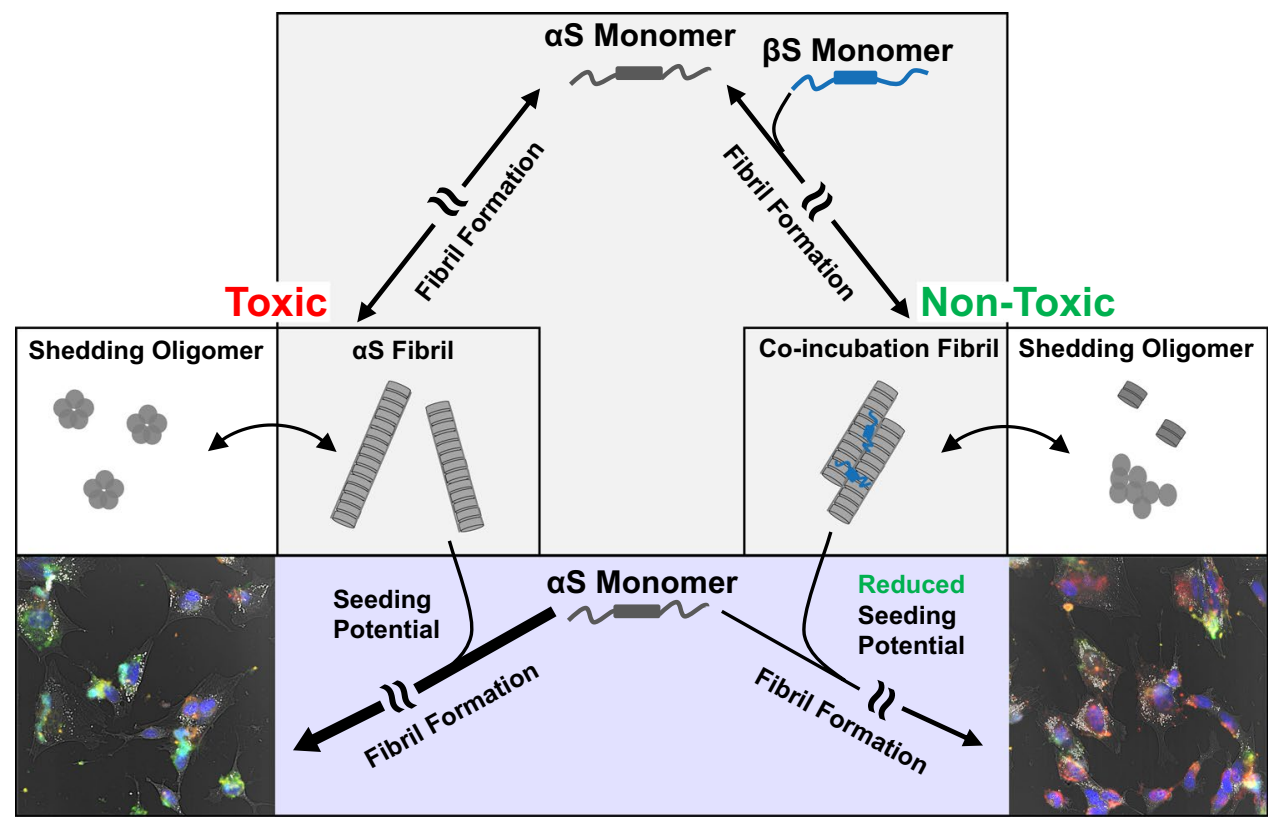

Figure 6. $\alpha S$ and $\alpha S / \beta S$ fibril toxicities and seeding potentials. $\alpha S$ aggregates and misfolds along a nucleationdependent fibril formation pathway, generating various oligomeric species before finally adopting a characteristic repeating cross-beta amyloid fibril structure. When $\alpha \mathrm{S}$ aggregates on its own (left pathway), the resulting fibrils are toxic to cultured human neuroblastoma cells, and the oligomeric species that shed from these fibrils are also toxic to cells. However, if $\alpha \mathrm{S}$ is co-incubated with $\beta S$ and allowed to aggregate (right pathway), then the resulting fibrils are no longer toxic to cells, and oligomer species that shed from these fibrils are also non-toxic. The $\alpha \mathrm{S}$ and $\alpha \mathrm{S} / \beta \mathrm{S}$ fibrils also display differential seeding capacities (confocal images, bottom). $\alpha S$ fibrils are able to efficiently seed amyloid formation, while co-incubated $\alpha \mathrm{S} / \beta \mathrm{S}$ fibrils have reduced propensity to seed further aggregation, as evidenced by the difference in green intensity in confocal images.

morphologies, while oligomers shed from $\alpha \mathrm{S} / \beta \mathrm{S}$ fibrils tend to adopt short proto-fibril morphologies with some larger globular species also present (Fig. 5b). We next measured the toxicity of the shed oligomers in SH-SY5Y cells. After a 48 hour period of incubation with shed oligomers from either $\alpha S$ or $\alpha S / \beta S$ fibrils, we found that oligomers shed from $\alpha$ S reduced cell viability by $17 \%$ compared to the untreated cells and cells treated with monomeric $\alpha S$, whereas oligomers shed from $\alpha \mathrm{S} / \beta \mathrm{S}$ did not (Fig. $5 \mathrm{c}$ ). We also assessed the ability of shed oligomers to seed further aggregation in cells, using confocal fluorescence microscopy. Compared with cells treated with monomeric $\alpha S$ (Fig. 5d, bottom row), cells treated with oligomers shed from $\alpha$ S fibrils showed an increase in anti-synuclein antibody fluorescence of $1.6 \times$ (Fig. $5 \mathrm{~d}$, top row), while cells treated with oligomers shed from $\alpha \mathrm{S} / \beta \mathrm{S}$ fibrils showed an increase of $1.3 \times$ (Fig. $5 \mathrm{~d}$, middle row). ThioS staining indicates that amyloid formation increased by $1.6 \times$ in cells treated with oligomers shed from $\alpha$ S fibrils and by $1.3 \times$ in cells treated with oligomers shed from $\alpha S / \beta S$ fibrils.

\section{Discussion}

Amyloid fibrils of $\alpha \mathrm{S}$ are key pathologic features of $\mathrm{PD}$ and have been recognized as contributing to the progression of the disease. These fibrils are thought to contribute to cellular toxicity through their ability to seed further aggregation of endogenous $\alpha \mathrm{S}$, and the ability of the fibrils to "shed" oligomer and protofibril species that may be toxic. The $\alpha \mathrm{S}$ fibrils studied in this work should be distinguished from fibrils that are contained within aggresome-like LBs. Fibrils that are formed as the end product of the aggregation pathway of $\alpha \mathrm{S}$ (either in vivo or in vitro), and are not yet collected into LBs, exist in a dynamic equilibrium with oligomers, as evidenced by the ability of fibrils to "shed" smaller molecular species ${ }^{18,63}$. Here we have demonstrated that $\alpha S$ fibrils formed in the presence of the natural inhibitor $\beta S$, while maintaining similar core structures as $\alpha$ S fibrils alone, exhibit reduced toxicity to neuroblastoma cells, reduced seeding properties, and are in dynamic equilibrium with oligomers that also share reduced toxicity and seeding (Fig. 6).

We have utilized ssNMR and the changes in ${ }^{13} \mathrm{C}$ chemical shifts to probe how the core residues and dynamics of $\alpha$ S fibrils and co-incubated $\alpha S / \beta S$ fibrils differ from one another. While we have not yet completed a full assignment and structure determination of our fibrils, ${ }^{13} \mathrm{C}$ chemical shifts are very sensitive reporters of amino acid type and secondary structure ${ }^{64}$. The cross-polarization based ssNMR experiments used in this work preferentially detect molecules and domains that are rigid, and dynamic and disordered domains are not detected. The dynamic regions of $\alpha S$ fibrils are the very $\mathrm{N}$-terminal (residues 1-43) and C-terminal (residues 97-140) domains; what we detect in our spectra are the relatively rigid core of the amyloid fibrils (residues $\sim 44-96$ ). The ${ }^{13} \mathrm{C}$ resonances observed in our spectra show characteristic $\beta$-sheet chemical shifts. Comparison of our spectra with the chemical shift lists (BMRB Entry 25518) and spectra reported by Rienstra and coworkers ${ }^{41}$, who have previously determined the core fibril structure of full-length $\alpha \mathrm{S}$ by ssNMR, show relatively good agreement (Fig. 2c). Since we 
have prepared our fibrils in a similar manner to those used for the published $\alpha S$ fibril structure ${ }^{41}$, we can reasonably assume that our fibrils adopt a similar core structure. $\alpha S$ has a 140 amino acid primary sequence generally described by 3 domains: a 60 residue polyampholyte $\mathrm{N}$-terminal domain, a 35 residue hydrophobic NAC domain, and a 45 residue highly negatively-charged polyelectrolyte $\mathrm{C}$-terminal domain. Lysine and threonine (Fig. 3e) are almost exclusively located in the N-terminal and NAC regions of the $\alpha \mathrm{S}$ sequence, where they are clustered into imperfect KTKEGV repeats. These two regions form the "Greek-key" motif of the $\alpha \mathrm{S}$ fibril core structures, roughly spanning residues 44-96. Of particular note is the preNAC domain (44-60), which makes up the packing interface between two $\alpha S$ protofilaments ${ }^{13,39,40}$, and contains two full KTK repeats.

While the overall fold of the core residues does not change between $\alpha \mathrm{S}$ and $\alpha \mathrm{S} / \beta \mathrm{S}$ fibrils, and is similar to previously determined $\alpha \mathrm{S}$ structures, based on the assignments of the Thr and Lys cross-peaks in our $2 \mathrm{D}^{13} \mathrm{C}-{ }^{13} \mathrm{C}$ spectra of the co-incubated $\alpha S / \beta S$ fibril we do find changes to these residues that make up the preNAC domain's final two KTKEGV repeats. First, from the loss of intensity in our $2 \mathrm{D}$ spectra and measurements of $\mathrm{T}_{1 \rho}$ relaxation, we have found an increase in the local dynamics of the Thr and Lys residues of co-incubated $\alpha S / \beta S$ fibrils relative to $\alpha S$ fibrils alone. Second, we observed that the water accessibility of the Lys residues increases in the co-incubated $\alpha S / \beta S$ fibrils relative to $\alpha S$ fibrils. Our ssNMR measurements of the fibril core structure (residues 44-96), and residue dynamics and water-accessibility highlight that $\beta S$ does not perturb the core structure of $\alpha S$ fibrils, but instead may have an impact on the residues at the protofilament packing interface.

Recent structures of $\alpha S$ fibrils determined by cryo-electron microscopy have begun to show the importance of protofilament packing to the observed differences in fibril polymorphism, rather than a change in the conformation of the protofilament core ${ }^{13,39,40}$. Our AFM images indicate that the primary distinctions between $\alpha \mathrm{S}$ and $\alpha \mathrm{S} / \beta \mathrm{S}$ fibrils lie in the average height and length, although these parameters are widely distributed (Fig. 1c,d). The more subtle distinction lies with the change in polymorph composition of the fibrils: $\alpha S$ fibrils show a mixture of twisted and straight polymorphs (Fig. 1a) while $\alpha \mathrm{S} / \beta \mathrm{S}$ fibrils appear to only have straight polymorphs (Fig. 1b). These observations suggest that $\beta S$ modulates the packing of the protofilaments in the mature fibril, and is less likely to be incorporated into the cross-beta structure of the individual protofilament. A change in protofilament packing is also supported by the proteinase K digestion profiles, which indicate that $\alpha \mathrm{S} / \beta \mathrm{S}$ fibrils are more easily accessible to cleavage by proteinase $\mathrm{K}$, suggesting that the co-incubated $\alpha \mathrm{S} / \beta \mathrm{S}$ fibrils might be more susceptible to degradation in vivo (Fig. 3d). These profiles resemble those from previous work by Miake and coworkers, who established that proteinase $\mathrm{K}$ digestion of $\alpha \mathrm{S}$ preferentially cleaves the $\mathrm{N}$ - and C-terminal portions of $\alpha \mathrm{S}$ and leaves the fibril core from residues $31-109$ intact $^{65}$. In addition, our results demonstrate that while $\alpha S$ fibrils are indeed toxic to neuroblastoma cells (Fig. 4a), co-incubated $\alpha \mathrm{S} / \beta \mathrm{S}$ fibrils are not (Fig. $4 \mathrm{a}$ ) and have a lesser tendency to cause the formation of synuclein aggregates and amyloid species compared with $\alpha \mathrm{S}$ fibrils (Fig. 4b). Therefore, co-incubation of $\beta S$ with $\alpha S$ results in fibrils that are not toxic to cells and have reduced ability to seed further aggregation in a cellular environment. The reduced seeding ability also suggests that $\beta S$ interferes with the ability of $\alpha S$ fibrils to catalyze secondary nucleation processes on the fibril surface, proposed previously by Knowles and coworkers ${ }^{66}$. Taken together with our earlier results, these results again suggest that $\beta$ S is associated with the $\mathrm{N}$-terminal domain and protofilament packing interface along $\alpha \mathrm{S}$ fibrils, inducing more dynamic flexibility in the $\mathrm{N}$-terminal portions of the $\alpha \mathrm{S}$ fibrils, and may suggest that $\mathrm{N}$-terminal domain dynamics and packing may play a role in this seeding process.

We have also found that $\alpha$ S fibrils are less thermostable (Fig. S2) and shed primarily small globular and amorphous oligomers (Fig. 5a), while co-incubated $\alpha S / \beta S$ fibrils are more thermostable (Fig. S1) and shed primarily short proto-fibril aggregates (Fig. 5b). The proto-fibril species shed from $\alpha S / \beta S$ fibrils also show reduced seeding propensity compared to the small globular and amorphous $\alpha \mathrm{S}$ oligomer species (Fig. 5d). This finding suggests that the dynamic equilibrium is shifted away from the formation of small toxic oligomers towards less toxic proto-fibrils in the presence of $\beta S$ (Fig. 5 c). Taken together in the context of our previous observations, the protofilament-packing of co-incubated $\alpha S / \beta S$ fibrils appears to be more stable than $\alpha$ S fibrils, while increasing the local dynamics of the $\mathrm{N}$-terminal domain. This results in a reduced capacity for seeding and shedding of toxic oligomeric species.

$\beta$ S has previously been identified in studies of transgenic mice as a natural anti-Parkinsonian factor which has the ability to reduce $\alpha S$ inclusion formation ${ }^{30}$. Yet, even though it reduces $\alpha S$ positive inclusions, it does not completely abolish the formation of $\alpha S$ fibrils. We propose that the role of $\beta S$ as an inhibitor is multifaceted, influencing $\alpha S$ aggregation at multiple points along its fibril-formation pathway. In the earliest stages of $\alpha S$ aggregation, $\beta S$ can stabilize $\alpha S$ in $\alpha S-\beta S$ heterodimers ${ }^{35}$, which help to slow down the conversion of $\alpha S$ into higher order aggregates. As $\alpha S$ continues to aggregate, $\beta S$ has been found to stabilize and eliminate the formation of toxic oligomers $^{67,68}$. In this work, we have now shown that in the last stage of $\alpha$ S aggregation, co-incubation with $\beta S$ minimizes the toxicity and seeding ability of $\alpha S$ fibrils, and furthermore alters the fibril-oligomer equilibrium. Our findings demonstrate that $\beta S$ can reduce the effects of toxic $\alpha S$ fibrils in cells without changing the core structure of $\alpha S$ fibrils, and provide insight into how the dynamics and the surface of these fibrils may directly contribute to their toxicity and seeding ability. The multi-pronged targeting of $\alpha S$ by $\beta S$ highlights the potential of $\beta S$ as a lead for the future design of inhibitors that provide therapeutic intervention in synucleinopathies at multiple stages of $\alpha \mathrm{S}$ aggregation.

The misfolding and aggregation of endogenous $\alpha \mathrm{S}$ monomers due to seeding by fibrils is believed to be critical to the progression of synucleinopathies. The mechanism by which mature $\alpha S$ fibrils seed further aggregation is believed to proceed by surface-mediated secondary nucleation ${ }^{69-71}$, where the surface properties of $\alpha S$ fibrils govern their interaction with endogenous $\alpha \mathrm{S}$ monomers and template further aggregation. The exact details of how additional $\alpha \mathrm{S}$ monomers undergo templated conversion are not yet known, but the present work provides some clues. The recent cryo-EM structures of $\alpha \mathrm{S}$ fibrils show that a steric-zipper motif in the N-terminal domain mediates the interface between two protofilaments and stabilizes the mature fibril morphology ${ }^{13,40}$. Our results show that co-incubated $\alpha \mathrm{S} / \beta \mathrm{S}$ fibrils have increased dynamics and water accessibility of residues in the $\mathrm{N}$-terminal 
domain, particularly in the KTKEVG repeats in the preNAC region, and allow for enhanced protease degradation of the fibril, suggesting that the protofilament interface may be altered and more dynamic. These observations also highlight the importance of dynamics in mediating the seeding ability of $\alpha \mathrm{S}$ fibrils: increased dynamics of the $\mathrm{N}$-terminal domain may lead to reduced seeding, as secondary nucleation may necessitate a rigid $\mathrm{N}$-terminal domain for proper templating of $\alpha S$ aggregation. Our results suggest that enhancing amyloid fibril dynamics at templating domains may be an approach for future therapeutic intervention for neurodegenerative diseases.

\section{Materials and Methods}

Protein expression and purification. Expression of $\mathrm{N}$-terminally acetylated human $\alpha \mathrm{S}$ and $\beta \mathrm{S}$ proteins was performed via co-expression with pNatB plasmid (Addgene \#53613) in E. coli BL21(DE3) cells, and protein purification was performed as described previously ${ }^{72}$. Uniformly ${ }^{13} \mathrm{C},{ }^{15} \mathrm{~N}$ isotopically labeled $\alpha \mathrm{S}$ for ssNMR experiments was expressed in M9 minimal media supplemented with ${ }^{13} \mathrm{C}$-glucose and ${ }^{15} \mathrm{~N}$-ammonium chloride as the sole carbon and nitrogen sources, respectively. Protein molecular weight and purity were assessed by ESI-MS, and stored at $-20^{\circ} \mathrm{C}$ as a lyophilized powder until use.

Fibril sample preparation. Lyophilized acetylated $\alpha \mathrm{S}$ or $\beta S$ was dissolved in $10 \mathrm{mM}$ PBS (pH 7.4), and large aggregates were removed by centrifuge filtration ( $50 \mathrm{kDa}$ MWCO, Millipore Sigma, St. Louis, MO). The dissolved protein was concentrated in $3 \mathrm{kDa}$ centrifuge units (Millipore Sigma, St. Louis, MO) to $1 \mathrm{mg} / \mathrm{mL}(\alpha \mathrm{S})$ or $3 \mathrm{mg} /$ $\mathrm{mL}(\beta S)$. To create fibrils, $100 \mathrm{uL}$ of each sample mixture was loaded into 96-well clear bottom plates (Corning, Corning, NY) with a single Teflon bead ( $3 \mathrm{~mm}$, Saint-Gobain N.A., Malvern PA). The plates were sealed with Axygen sealing tape (Corning, Corning, NY) and shaken at $600 \mathrm{rpm}$ and $37^{\circ} \mathrm{C}$ in a POLARstar Omega fluorimeter (BMG Labtech, Cary, NC). Fibrils were allowed to form for at least 72 hours. Samples used for AFM, ESI-MS, ssNMR, and cell toxicity and shedding experiments were collected by centrifugation at $14 \mathrm{k} \mathrm{rpm}$ for 2 hours, and washed through multiple rounds of re-suspension in $10 \mathrm{mM}$ PBS (pH 7.4) and centrifugation at $14 \mathrm{k} \mathrm{rpm} \mathrm{for}$ 2 hours in order to remove residual soluble and non-fibrillar components.

Preparation of oligomer species shed from fibrils. Fibril samples were re-suspended in $1 \mathrm{~mL}$ of $10 \mathrm{mM}$ PBS ( $\mathrm{pH} 7.4$ ) and incubated at $37^{\circ} \mathrm{C}$ for $72 \mathrm{~h}$, followed by removal of mature fibrils by using $0.22 \mu \mathrm{m}$ filter (Millipore Sigma, St. Louis, MO). Samples were concentrated with $3 \mathrm{kDa}$ centrifuge units (Millipore Sigma, St. Louis, MO), and protein concentration was measured using a bicinchoninic acid (BCA) assay (Thermo Scientific, Waltham, MA).

Proteinase $\mathrm{K}$ digestion. Fibrils at a concentration of $1 \mathrm{mg} / \mathrm{mL}$ were incubated with various concentrations $(0.1,0.5,1.0,2.0,5.0 \mu \mathrm{g} / \mathrm{mL})$ of proteinase K (Sigma Aldrich, St. Louis, MO) in $10 \mathrm{mM} \mathrm{PBS}(\mathrm{pH} 7.4)$ at $37^{\circ} \mathrm{C}$ for $1 \mathrm{~h}$. The digestion reaction was quenched by the addition of a 1200:1 molar excess of phenylmethane sulfonyl fluoride (Sigma Aldrich, St. Louis, MO) followed by the addition of $2 \mathrm{M}$ guanidine thiocyanate (Sigma Aldrich, St. Louis, MO) and incubation at room temperature for $4 \mathrm{~h}$. The results of the degradation reaction were mixed with 4x SDS-PAGE loading buffer (Invitrogen, Carlsbad, CA), loaded onto precast gels (Bio-Rad, Hercules, CA), and run at $120 \mathrm{~V}$ for $50 \mathrm{~min}$.

Atomic Force Microscopy (AFM). Samples $(20 \mu \mathrm{L})$ were placed onto freshly cleaved mica (Ted Pella Inc., Redding, CA) and incubated for $15 \mathrm{~min}$ at room temperature, followed by 3 washes of $200 \mu \mathrm{L}$ each deionized water as described previously ${ }^{34}$. All images were collected on a NX-10 instrument (Park Systems, Suwon, South Korea) using non-contact mode tips (PPP-NHCR, 42 N/m, $330 \mathrm{kHz}$; Nanosensors, Neuchatel, Switzerland). Image processing and analysis were carried out in the Gwyddion software package ${ }^{73}$.

Solid-State Nuclear Magnetic Resonance Experiments. All MAS ssNMR experiments were carried out on an Avance III HD $600 \mathrm{MHz}(14 \mathrm{~T})$ spectrometer (Bruker BioSpin, Billerica, MA) using a $1.6 \mathrm{~mm}$ triple resonance MAS probe (Phoenix NMR, Loveland, CO) tuned to ${ }^{1} \mathrm{H} /{ }^{13} \mathrm{C} /{ }^{15} \mathrm{~N}$ frequencies. Typical radiofrequency (rf) field strengths were $118 \mathrm{kHz}$ for ${ }^{13} \mathrm{C}, 74 \mathrm{kHz}$ for ${ }^{15} \mathrm{~N}$, and $100-145 \mathrm{kHz}$ for ${ }^{1} \mathrm{H} .{ }^{13} \mathrm{C}$ chemical shifts were referenced to the ${ }^{13} \mathrm{CH}_{2}$ signal of adamantane at $38.48 \mathrm{ppm}$ on the tetramethylsilane (TMS) scale, and ${ }^{15} \mathrm{~N}$ chemical shifts were referenced to the ${ }^{15} \mathrm{~N}$ signal of $\mathrm{N}$-acetylvaline at $122.0 \mathrm{ppm}$ on the liquid ammonia scale. All experiments utilized a MAS rate of $13.333 \mathrm{kHz}$, and sample temperature was controlled to $25^{\circ} \mathrm{C}$, unless otherwise noted. One-dimensional (1D) ${ }^{13} \mathrm{C}$ MAS spectra were recorded using a conventional cross-polarization (CP) sequence. Two-dimensional (2D) ${ }^{13} \mathrm{C}-{ }^{13} \mathrm{C}$ dipolar-assisted rotational-resonance (DARR) experiments ${ }^{74} \mathrm{utilized}$ a mixing period of $100 \mathrm{~ms}$. A 2D water-edited DARR ${ }^{50}$ experiment, with a DARR mixing period of $100 \mathrm{~ms}$, a $\mathrm{T}_{2}$-filter of $6 \mathrm{~ms}$, and a ${ }^{1} \mathrm{H}$ spin-diffusion period of either $3 \mathrm{~ms}$ or $100 \mathrm{~ms}$, was used to measure the water-protein ${ }^{1} \mathrm{H}$ spin diffusion differences between the two fibril samples. $2 \mathrm{D}^{15} \mathrm{~N}-{ }^{13} \mathrm{C}$ correlation spectra were measured using a REDOR-based pulse sequence ${ }^{75}$, utilizing a REDOR period of $1.35 \mathrm{~ms}$ to observe long range correlations. A standard Bruker $\mathrm{CP}$ based pulse sequence was used to measure the ${ }^{13} \mathrm{C}_{1 \rho}$ relaxation of the $\mathrm{Thr} C \beta^{49} . \mathrm{A}^{13} \mathrm{C} \omega_{1}$ field of $2.25 \omega_{\mathrm{r}}$ was applied on resonance with the Thr C $\beta(70 \mathrm{ppm})$ during the spin-lock period.

Analysis of fibril composition by ESI-MS. Mature fibril samples were dissolved in $4 \mathrm{M}$ guanidine hydrochloride overnight, then buffer exchanged with $50 \mathrm{mM}$ ammonium acetate with $0.1 \%$ formic acid. Samples were concentrated to $10 \mu \mathrm{M}$ for ESI-MS analysis.

Neuroblastoma cell culture. Human SH-SY5Y neuroblastoma cells (ATCC, Manassas, VA) were cultured in DMEM/F12 (GE Healthcare, Boston, MA) with 10\% fetal bovine serum (Gibco Co., Dublin, Ireland) and kept in a $37^{\circ} \mathrm{C}, 5 \% \mathrm{CO}_{2}$ humidified atmosphere. Before cell viability assays or immunocytochemistry, cells were plated 
into 96-well (Corning, Corning, NY) or 12-well plates (Cellvis, Mountain View, CA), and allowed to grow for $24 \mathrm{~h}$.

Cell viability MTS reduction assay. SH-SY5Y cells were treated with $1.3 \mu \mathrm{M}$ fibril $(24 \mathrm{~h}), 0.7 \mu \mathrm{M}$ shed species $(48 \mathrm{~h})$, or an equivalent concentration of monomer as a control ( $24 \mathrm{~h}$ or $48 \mathrm{~h}$ ). Cell viability was assessed by adding $20 \mu \mathrm{L}$ MTS per $100 \mu \mathrm{L}$ cell culture (Promega, USA) and incubating for $2.5 \mathrm{~h}$ at $37^{\circ} \mathrm{C}$, before measuring absorbance at $490 \mathrm{~nm}$.

Immunocytochemistry. SH-SY5Y cells were treated with $1.3 \mu \mathrm{M}$ fibril (24h), $0.7 \mu \mathrm{M}$ shed species (48 h), or an equivalent concentration of monomer as a control $(24 \mathrm{~h}$ or $48 \mathrm{~h}$ ). Cells were fixed with $10 \%$ formalin (Sigma Aldrich, St. Louis, MO) and permeabilized with 0.5\% Triton in PBS (Sigma Aldrich, St. Louis, MO). Cells were then blocked by incubation with 5\% Donkey Serum solution (Sigma Aldrich, St. Louis, MO) for 30 min at $37^{\circ} \mathrm{C}$. Cells were incubated with $0.01 \%$ thioflavin S (Acros Organics, Waltham, MA) for $10 \mathrm{~min}$ at $37^{\circ} \mathrm{C}$ and then washed with PBS, followed by incubation with purified mouse anti- $\alpha$-synuclein primary antibody (BD Biosciences, Franklin Lakes, NJ; Cat.\# 610786, RRID: AB_398107) at $4{ }^{\circ} \mathrm{C}$ overnight in the dark. Cells were washed with PBS 3 times and incubated with fluorophore-conjugated secondary antibody TRITC (Sigma Aldrich, St. Louis, MO; Cat.\# T5393, RRID: AB_261699) for $1 \mathrm{~h}$, then washed again 3 times with PBS. Cells were incubated with DAPI for $1.5 \mathrm{~min}$ at room temperature and then washed with PBS, to visual cell nuclei. All samples were imaged using a Zeiss LSM 780 confocal laser scanning microscope with 20x objective (Zeiss, Oberkochen, Germany), and images were processed using the Fiji distribution of Image ${ }^{76}$. To analyze the anti- $\alpha S$-antibody and ThioS stained fluorescence images in a more quantitative manner, Fiji was used to filter and threshold each channel based on the DAPI-stained nuclei to extract the raw intensity per cell for each channel. These intensities were then normalized by the intensity per cell of the monomer treated cells, to give the relative increase in intensity reported in the results section. DIC overlays of fluorescence images presented in Figs. $4 \mathrm{~b}$ and $5 \mathrm{~d}$ are shown in the SI (Fig. S9).

Preparation of ATTO-550 labelled fibrils. Fibrils were labeled with the fluorescent ATTO550-NHS-ester (ATTO-TEC GmbH) per the manufacturer's procedure. In brief, fibrils formed as described above were incubated with a $2 \mathrm{M}$ excess of ATTO550-NHS-ester in labeling buffer ( $\mathrm{pH} 8.3 \mathrm{PBS} /$ sodium bicarbonate solution) for 1 hour at room temperature. Conjugated ATTO550-fibrils were then separated from unreacted fluorophore by centrifugation at $16 \mathrm{k} \mathrm{rpm}$ for $30 \mathrm{~min}$ and resuspension of the ATTO550-fibril pellet in $\mathrm{pH}$ 7.4 PBS; this centrifugation/ resuspension wash was repeated twice.

Statistical analysis. All cell viability experiments were performed in triplicate, and each assay was repeated at least three times. Statistical significance was determined by one-way ANOVA with post-hoc Bonferonni analysis (GraphPad Prism).

Received: 15 February 2019; Accepted: 29 October 2019;

Published online: 26 November 2019

\section{References}

1. Tysnes, O.-B. \& Storstein, A. Epidemiology of Parkinson's disease. J. Nerual. Transm. 124, 901-905 (2017).

2. Ross, C. A. \& Poirier, M. A. Protein aggregation and neurodegenerative disease. Nat. Med. 10, S10-S17 (2004).

3. Vekrellis, K., Xilouri, M., Emmanouilidou, E., Rideout, H. J. \& Stefanis, L. Pathological roles of $\alpha$-synuclein in neurological disorders. Lancet Neurol. 10, 1015-1025 (2011).

4. Volpicelli-Daley, L. A. et al. Exogenous $\alpha$-synuclein fibrils induce Lewy body pathology leading to synaptic dysfunction and neuron death. Neuron 72, 57-71 (2011).

5. Pieri, L., Madiona, K., Bousset, L. \& Melki, R. Fibrillar $\alpha$-synuclein and huntingtin exon 1 assemblies are toxic to the cells. Biophys. J. 102, 2894-2905 (2012).

6. Bousset, L. et al. Structural and functional characterization of two $\alpha$-synuclein strains. Nat. Commun. 4, 2575 (2013).

7. Mammadova, N. et al. Accelerated accumulation of retinal $\alpha$-synuclein (pSer129) and tau, neuroinflammation and autophagic dysregulation in a seeded mouse model of Parkinson's disease. Neurobiol. Dis. 121, 1-16 (2019).

8. Freundt, E. C. et al. Neuron-to-neuron transmission of $\alpha$-synuclein fibrils through axonal transport. Ann. Neurol. 72, 517-524 (2012).

9. Longhena, F. et al. The contribution of $\alpha$-synuclein spreading to Parkinson's disease synaptopathy. Neural. Plast. 2017, 5012129 (2017).

10. Mao, X. et al. Pathological $\alpha$-synuclein transmission initiated by binding lymphocyte-activation gene 3. Science 353, aah3374 (2016).

11. Rodriguez, L., Marano, M. M. \& Tandon, A. Import and export of misfolded $\alpha$-synuclein. Front. Neurosci. 12, 344 (2018).

12. Yan, R. et al. Synergistic neuroprotection by coffee components eicosanoyl-5-hydroxytryptamide and caffeine in models of Parkinson's disease and DLB. Proc. Natl. Acad. Sci. USA In Press, https://doi.org/10.1073/pnas.1813365115 (2018).

13. Li, B. et al. Cryo-EM of full-length $\alpha$-synuclein reveals fibril polymorphs with a common structural kernel. Nat. Commun. 9, 3609 (2018).

14. Vilar, M. et al. The fold of $\alpha$-synuclein fibrils. Proc. Natl. Acad. Sci. USA 105, 8637-8642 (2008).

15. Peelaerts, W. et al. $\alpha$-Synuclein strains cause distinct synucleinopathies after local and systemic administration. Nature 522, 340-344 (2015).

16. Peng, C. et al. Cellular milieu imparts distinct pathological $\alpha$-synuclein strains in $\alpha$-synucleinopathies. Nature 557, 558-563 (2018).

17. Tipping, K. W. et al. $\mathrm{pH}$-induced molecular shedding drives the formation of amyloid fibril-derived oligomers. Proc. Natl. Acad. Sci USA 112, 5691-5696 (2015)

18. Cremades, N. et al. Direct observation of the interconversion of normal and toxic forms of $\alpha$-synuclein. Cell 149, 1048-1059 (2012).

19. Tarutani, A. et al. The effect of fragmented pathogenic $\alpha$-synuclein seeds on prion-like propagation. J. Biol. Chem. 291, 18675-18688 (2016).

20. Karpowicz, R. J. et al. Selective imaging of internalized proteopathic $\alpha$-synucelin seeds in primary neurons reveals mechanistic insight into transmission of synucleinopathies. J. Biol. Chem. 292, 13482-13497 (2017).

21. Holmes, B. B. et al. Heparan sulfate proteoglycans mediate internalization and propagation of specific proteopathic seeds. Proc. Natl. Acad. Sci. USA 110, E3138-E3147 (2013). 
22. Shrivastava, A. N. et al. $\alpha$-Synuclein assemblies sequester neuronal $\alpha 3-\mathrm{Na}^{+} / \mathrm{K}^{+}$-ATPase and impair $\mathrm{Na}^{+}$gradient. $E M B O J .34$, 2408-2423 (2015).

23. Freeman, D. et al. Alpha-synuclein induces lysosomal rupture and cathepsin dependent reactive oxygen species following endocytosis. PLoS ONE 8, e62143 (2013).

24. Flavin, W. P. et al. Endocytic vesicle rupture is a conserved mechanism of cellular invasion by amyloid proteins. Acta Neuropathol. 134, 629-653 (2017).

25. Samuel, F. et al. Effects of serine 129 phosphorylation on $\alpha$-synuclein aggregation, membrane association, and internalization. J. Biol. Chem. 291, 4374-4385 (2016).

26. Tanik, S. A., Schultheiss, C. E., Volpicelli-Daley, L. A., Brunden, K. R. \& Lee, V. M. Y. Lewy body-like $\alpha$-synuclein aggregates resist degradation and impair macroautophagy. J. Biol. Chem. 288, 15194-15210 (2013).

27. Abounit, S. et al. Tunneling nanotubes spread fibrillar $\alpha$-synuclein by intercellular trafficking of lysosomes. EMBO J. 35, 2120-2138 (2016).

28. Beyer, K., Ispierto, L., Latorre, P., Tolosa, E. \& Ariza, A. Alpha- and beta-synuclein expression in Parkison disease with and without dementia. J. Neruol. Sci. 310, 112-117 (2011).

29. Rockenstein, E. et al. Altered expression of the synuclein family mRNA in Lewy body and Alzheimer's disease. Brain Res. 914, 48-56 (2001).

30. Hashimoto, M., Rockenstein, E., Mante, M., Mallory, M. \& Masliah, E. $\beta$-Synuclein inhibits $\alpha$-synuclein aggregation: a possible role as an anti-parkinsonian factor. Neuron 32, 213-223 (2001).

31. Chartier, S. \& Duyckaerts, C. Is Lewy pathology in the human nervous system chiefly an indicator of neuronal protection or of toxicity? Cell Tissue Res. 373, 149-160 (2018).

32. Wakabayashi, K. et al. The Lewy body in Parkinson's disease and related neurodegenerative disorders. Mol. Neurobiol. 47, 495-508 (2013).

33. Williams, J. K. et al. Multi-pronged interactions underlie inhibition of $\alpha$-synuclein aggregation by $\beta$-synuclein. J. Mol. Biol. 430, 2360-2371 (2018).

34. Moriarty, G. M. et al. A pH-dependent switch promotes $\beta$-synuclein fibril formation via glutamate residues. J. Biol. Chem. 292, 16368-16379 (2017).

35. Janowska, M. K., Wu, K. P. \& Baum, J. Unveiling transient protein-protein interactions that modulate inhibition of alpha-synuclein aggregation by beta-synuclein, a pre-synaptic protein that co-localizes with alpha-synuclein. Sci. Rep. 5, 15164 (2015).

36. Uversky, V. N. et al. Biophysical properties of the synucleins and their propensities to fibrillate: inhibition of $\alpha$-synuclein assembly by $\beta$ - and $\gamma$-synucleins. J. Biol. Chem. 277, 11970-11978 (2002).

37. Lashuel, H. A., Overk, C. R., Oueslati, A. \& Masliah, E. The many faces of $\alpha$-synulcein: From structure and toxicity to therapeutic target. Nat. Rev. Neurosci. 14, 38-48 (2013).

38. Birol, M., Wojcik, S. P., Miranker, A. D. \& Rhoades, E. Identification of N-linked glycans as specific mediators of neuronal uptake of acetylated $\alpha$-synuclein. PLoS Biol. 17, e3000318 (2019).

39. Li, Y. et al. Amyloid fibril structure of $\alpha$-synuclein determined by cryo-electron microscopy. Cell Res. 28, 897-903 (2018).

40. Guerrero-Ferreira, R. et al. Cryo-EM structure of alpha-synuclein fibrils. eLife 7, e36402 (2018).

41. Tuttle, M. D. et al. Solid-state NMR structure of a pathogenic fibril of full-length human $\alpha$-synuclein. Nat. Struct. Mol. Biol. 23, 409-415 (2016).

42. Ulrich, E. L. et al. BioMagResBank. Nuc. Acids Res. 36, D402-D408 (2008).

43. Comellas, G. et al. Structured regions of $\alpha$-synuclein fibrils include the early-onset Parkinson's disease mutation sites. J. Mol. Biol. 411, 881-895 (2011).

44. Gath, J. et al. Solid-state NMR sequential assignments of $\alpha$-synuclein. Biomol. NMR Assign. 6, 51-55 (2012).

45. Comellas, G., Lemkau, L. R., Zhou, D. H., George, J. M. \& Rienstra, C. M. Structural intermediates during $\alpha$-synuclein fibrillogenesis on phospholipid vesicles. J. Am. Chem. Soc. 134, 5090-5099 (2012).

46. Zhou, D. H. et al. Solid-state NMR analysis of membrane proteins and protein aggregates by proton detected spectroscopy. J. Biomol. NMR 54, 291-305 (2012).

47. Verasdonck, J. et al. Further exploration of the conformational space of $\alpha$-synuclein fibrils: Solid-state NMR assignment of a highpH polymorph. Biomol. NMR Assign. 10, 5-12 (2016).

48. Barclay, A. M., Dhavale, D. D., Courtney, J. M., Kotzbauer, P. T. \& Rienstra, C. M. Resonance assignements of an $\alpha$-synuclein fibril prepared in Tris buffer at moderate ionic strength. Biomol. NMR Assign. 12, 195-199 (2018).

49. Krushelnitsky, A. et al. Expanding the frequency range of the solid-state $\mathrm{T}_{1 \rho}$ experiment for heteronuclear dipolar relaxation. Solid State Nucl. Magn. Reson. 22, 423-438 (2002).

50. Andronesi, O. C. et al. Characterization of Alzheimer's-like paired helical filaments from the core domain of tau protein using solidstate NMR spectroscopy. J. Am. Chem. Soc. 130, 5922-5928 (2008)

51. Williams, J. K. \& Hong, M. Probing membrane protein structure using water polarization transfer solid-state NMR. J. Magn. Reson. 247, 118-127 (2014).

52. Williams, J. K., Tietze, D., Lee, M., Wang, J. \& Hong, M. Solid-state NMR investigation of the conformation, proton conduction, and hydration of the influenza B virus M2 transmembrane proton channel. J. Am. Chem. Soc. 138, 8143-8155 (2016).

53. Elkins, M. R. et al. Structural polymorphism of Alzheimer's beta-amyloid fibrils as controlled by an E22 switch: A solid-state NMR study. J. Am. Chem. Soc. 138, 9840-9852 (2016).

54. Wang, T., Jo, H., DeGrado, W. F. \& Hong, M. Water distribution, dynamics and interactions with Alzheimer's beta-amyloid fibrils investigated by solid-state NMR. J. Am. Chem. Soc. 139, 6242-6252 (2017).

55. Gelenter, M. D. et al. The peptide hormone glucagon forms amyloid fibrils with two coexisting $\beta$-strand conformations. Nat. Struct. Mol. Biol. 26, 592-598 (2019).

56. Smith, D. M. Could a common mechanism of protein degradation impairment underlie many neurodegenerative diseases? J. Exp. Neurosci. 12, 1179069518794675 (2018).

57. Opazo, F., Krenz, A., Heermann, S., Schulz, J. B. \& Falkenburger, B. H. Accumulation and clearance of $\alpha$-synuclein aggregates demonstrated by time-lapse imaging. J. Neurochem. 106, 529-540 (2008).

58. Vilchez, D., Saez, I. \& Dillin, A. The role of protein clearance mechanisms in organismal ageing and age-related diseases. Nat. Commun. 5, 5659 (2014)

59. Luth, E. S., Stavrovskaya, I. G., Bartels, T., Kristal, B. S. \& Selkoe, D. J. Soluble, prefibrillar $\alpha$-synuclein oligomers promote complex I-dependent, $\mathrm{Ca}^{2+}$-induced mitochondrial dysfunction. J. Biol. Chem. 289, 21490-21507 (2014).

60. Polinski, N. K. et al. Best practices for generating and using alpha-synuclein pre-formed fibrils to model Parkinson's disease in rodents. J. Parkinsons Dis. 8, 303-322 (2018).

61. Xue, W. F. et al. Fibril fragmentation enhances amyloid cytotoxicity. J Biol Chem 284, 34272-82 (2009).

62. Tanaka, M. et al. Aggresomes formed by $\alpha$-synuclein and synphilin-1 are cytoprotective. J. Biol. Chem. 279, 4625-4631 (2004).

63. Carulla, N. et al. Molecular recycling within amyloid fibrils. Nature 436, 554-558 (2005).

64. Fritzsching, K. J., Hong, M. \& Schmidt-Rohr, K. Conformationally selective multidimensional chemical shift ranges in proteins from a PACSY database purged using intrinsic quality criteria. J. Biomol. NMR 64, 115-130 (2016).

65. Miake, H., Mizusawa, H., Iwatsubo, T. \& Hasegawa, M. Biochemical characterization of the core structure of $\alpha$-synuclein filaments. J. Biol. Chem. 277, 19213-19219 (2002). 
66. Brown, J. W. et al. Beta-Synuclein suppresses both the initiation and amplification steps of alpha-synuclein aggregation via competitive binding to surfaces. Sci Rep 6, 36010 (2016).

67. Israeli, E. \& Sharon, R. Beta-synuclein occurs in vivo in lipid-associated oligomers and forms hetero-oligomers with alpha-synuclein. J Neurochem 108, 465-74 (2009).

68. Leitao, A., Bhumkar, A., Hunter, D. J. B., Gambin, Y. \& Sierecki, E. Unveiling a Selective Mechanism for the Inhibition of $\alpha-S y n u c l e i n$ Aggregation by $\beta$-Synuclein. Int. J. Mol. Sci. 19, 334 (2018).

69. Tornquist, M. et al. Secondary nucleation in amyloid formation. Chem. Commun. 54, 8667-8684 (2018).

70. Gaspar, R. et al. Secondary nucleation of monomers on fibril surface dominates $\alpha$-synuclein aggregation and provides autocatalytic amyloid amplification. Q. Rev. Biophys. 50, e6 (2017).

71. Buell, A. K. et al. Solution conditions determine the relative importance of nucleation and growth processes in $\alpha$-synuclein aggregation. Proc. Natl. Acad. Sci. USA 111, 7671-7676 (2014).

72. Kang, L. et al. $\mathrm{N}$-terminal acetylation of $\alpha$-synuclein induces increased transient helical propensity and decreased aggregation rates in the intrinsically disordered monomer. Prot. Sci. 21, 911-917 (2012).

73. Necas, D. \& Kapetek, P. Gwyddion: an open-source software for SPM data analysis. Cent. Eur. J. Phys. 10, 181-188 (2012).

74. Takegoshi, K., Makamura, S. \& Terao, T. ${ }^{13} \mathrm{C}-{ }^{-1} \mathrm{H}$ dipolar-assisted rotational resonance in magic-angle spinning NMR. Chem. Phys. Lett. 344, 631-637 (2001).

75. Hong, M. \& Griffin, R. G. Resonance assignments for solid peptides by dipolar-mediated ${ }^{13} \mathrm{C} /{ }^{15} \mathrm{~N}$ correlation solid-state NMR. J. Am. Chem. Soc. 120, 7113-7114 (1998).

76. Schindelin, J. et al. Fiji: an open-source platform for biological-image analysis. Nat. Methods 9, 676-682 (2012).

\section{Acknowledgements}

This work was supported by National Institutes of Health (NIH) Grant GM110577 (J.B.). M.M.M. is the William Dow Lovett Professor of Neurology and is funded by NIH grants NS096032, AT006868, NS073994, and NS101134, the Michael J. Fox Foundation for Parkinson's Research, and the American Parkinson Disease Association.

\section{Author contributions}

J.B. and M.M.M. conceived the study. X.Y. expressed and purified protein, and prepared fibril and oligomer samples for all experiments. X.Y. performed the proteinase K assay, AFM and ESI-MS experiments. X.Y. and R.Y. designed and carried out the SH-SY5Y cell culture, MTS assay, and immunocytochemistry experiments. J.K.W. designed and performed the solid-state NMR experiments. X.Y., J.K.W., M.M.M. and J.B. analyzed the data and wrote the manuscript. All authors reviewed, edited and approved the manuscript.

\section{Competing interests}

The authors declare no competing interests.

\section{Additional information}

Supplementary information is available for this paper at https://doi.org/10.1038/s41598-019-54063-8.

Correspondence and requests for materials should be addressed to J.B.

Reprints and permissions information is available at www.nature.com/reprints.

Publisher's note Springer Nature remains neutral with regard to jurisdictional claims in published maps and institutional affiliations.

Open Access This article is licensed under a Creative Commons Attribution 4.0 International License, which permits use, sharing, adaptation, distribution and reproduction in any medium or format, as long as you give appropriate credit to the original author(s) and the source, provide a link to the Creative Commons license, and indicate if changes were made. The images or other third party material in this article are included in the article's Creative Commons license, unless indicated otherwise in a credit line to the material. If material is not included in the article's Creative Commons license and your intended use is not permitted by statutory regulation or exceeds the permitted use, you will need to obtain permission directly from the copyright holder. To view a copy of this license, visit http://creativecommons.org/licenses/by/4.0/.

(c) The Author(s) 2019 